PHYSICAL REVIEW D 78, 064010 (2008)

\title{
Cosmological perturbations from stochastic gravity
}

\author{
Albert Roura \\ Theoretical Division, T-8, Los Alamos National Laboratory, M.S. B285, Los Alamos, New Mexico 87545, USA \\ Enric Verdaguer \\ Departament de Física Fonamental and Institut de Ciències del Cosmos, Universitat de Barcelona, Avinguda Diagonal 647, \\ 08028 Barcelona, Spain
}

(Received 13 September 2007; published 2 September 2008)

\begin{abstract}
In inflationary cosmological models driven by an inflaton field the origin of the primordial inhomogeneities which are responsible for large-scale structure formation are the quantum fluctuations of the inflaton field. These are usually calculated using the standard theory of cosmological perturbations, where both the gravitational and the inflaton fields are linearly perturbed and quantized. The correlation functions for the primordial metric fluctuations and their power spectrum are then computed. Here we introduce an alternative procedure for calculating the metric correlations based on the Einstein-Langevin equation which emerges in the framework of stochastic semiclassical gravity. We show that the correlation functions for the metric perturbations that follow from the Einstein-Langevin formalism coincide with those obtained with the usual quantization procedures when the scalar field perturbations are linearized. This method is explicitly applied to a simple model of chaotic inflation consisting of a Robertson-Walker background, which undergoes a quasi-de Sitter expansion, minimally coupled to a free massive quantum scalar field. The technique based on the Einstein-Langevin equation can, however, deal naturally with the perturbations of the scalar field even beyond the linear approximation, as is actually required in inflationary models which are not driven by an inflaton field, such as Starobinsky's trace-anomaly driven inflation or when calculating corrections due to nonlinear quantum effects in the usual inflaton driven models.
\end{abstract}

DOI: 10.1103/PhysRevD.78.064010

PACS numbers: 04.62.+v, 03.65.Sq, 05.40.-a

\section{INTRODUCTION}

Inflation has become the paradigm for our understanding of the origin of the primordial inhomogeneities which are responsible for large-scale cosmic structure. The typical inflationary scenario assumes a period of accelerated expansion in the early Universe, usually driven by a scalar inflaton field, which provides a natural explanation for the homogeneity, isotropy, and flatness problems of the standard big-bang cosmology [1-6]. The generation of structure is explained by the back-reaction effect of the quantum fluctuations of the inflaton field on the gravitational field which translate, after quantization, into nontrivial twopoint correlation functions of the primordial gravitational fluctuations. These correlations give an approximate Harrison-Zeldovich spectrum for large scales [7-12]. The remarkable success of this scenario to explain the observed anisotropies of the cosmic microwave background [13-16] is today the most compelling reason which supports the inflationary paradigm $[17,18]$, in spite of some interpretational problems such as the transition from quantum to classical fluctuations [19-26].

Semiclassical gravity is a mean field approximation that describes the interaction of quantum matter fields with the gravitational field, which is treated as a classical geometry, and provides a suitable framework for the study of macroscopic black holes as well as scenarios in the early Universe after the Planck time. In particular it accommo- dates the different inflationary models. The key equation in semiclassical gravity is the semiclassical Einstein equation where the expectation value of the stress tensor operator of the quantum matter fields is the source of the spacetime metric. In cosmology this is usually assumed to be a spatially homogeneous and isotropic Robertson-Walker spacetime. However, since this theory relies only on the expectation value, it completely misses the fluctuations of the stress tensor operator. Thus, when the back reaction of the inhomogeneous fluctuations of the inflaton field around the homogeneous background are relevant, as in the generation of primordial inhomogeneities, the semiclassical equation is insufficient.

In recent years a stochastic semiclassical gravity (or stochastic gravity) approach has emerged as an extension of semiclassical gravity which accounts for the quantum fluctuations of the stress tensor [27,28]. These fluctuations are characterized by the noise kernel, which is defined as the symmetrized two-point quantum correlation function of the stress tensor operator. The extension is based on the so-called Einstein-Langevin equation, which is a stochastic equation for the linearized gravitational perturbations around a semiclassical background. A Gaussian stochastic source with a correlation function determined by the noise kernel is the key ingredient of this equation. From the solutions of the Einstein-Langevin equation, the two-point correlation functions for the metric perturbations can be obtained. 
Stochastic gravity provides an alternative framework to study the generation of primordial inhomogeneities in inflationary models. Besides the interest of the problem in its own right, there are also other reasons that make this problem worth discussing from the point of view of stochastic gravity. The Einstein-Langevin equation is not restricted by the use of linearized perturbations of the inflaton field. This may not be very important for inflationary models which are driven by an inflaton field which takes a nonzero expectation value, because the linear perturbations will give the leading contribution; although the need to consider higher-order corrections (from one-loop contributions) has recently been emphasized [29,30] (see also [31]). These contributions are in any case important in models such as Starobinsky's trace-anomaly driven inflation [32], which rely on conformally-coupled scalar fields with a vanishing expectation value. The corresponding Einstein equation is quadratic in these fields and the linear approximation becomes trivial.

In this paper we prove that the usual quantization for linear perturbations of both the metric and the inflaton field is equivalent to using the Einstein-Langevin equation when the latter is restricted to linearized inflaton perturbations, in the sense that the same results for the relevant correlation functions of the metric perturbations are obtained. ${ }^{1}$

The plan of the paper is the following. In Sec. II a brief description of stochastic gravity is given. This is done in an axiomatic way by showing that the semiclassical Einstein equation can be consistently generalized in a perturbative way by including a Gaussian stochastic source with vanishing expectation value defined through the noise kernel. The dynamical equation for the metric perturbations is the Einstein-Langevin equation. An alternative derivation of this equation, reviewed in Appendix $\mathrm{C}$, is based on the influence functional method due to Feynman and Vernon, which is generally used to describe the dynamics of open quantum systems, i.e., systems interacting with an environment $[34,35]$. Here the gravitational field plays the role of the system and the quantum matter fields play the role of the environment.

In Sec. III we discuss the linearized perturbations around a cosmological Robertson-Walker background coupled to a free massive scalar field minimally coupled to the curvature. This corresponds to the simplest model of chaotic inflation. The linearized Einstein-Langevin equation is then used to obtain an expression for the correlation function of the scalar-type metric perturbations. We concentrate on metric perturbations of scalar type because they are

\footnotetext{
${ }^{1}$ Although the equivalence between a stochastic description based on a Langevin equation and the results obtained with standard quantization methods has been exactly shown for linear open quantum systems [33], those results cannot be directly applied to the problem of linearized cosmological perturbations because the existing gauge freedom and dynamical constraints in the latter case play a nontrivial role.
}

the only ones that couple to the inflaton perturbations in the linear approximation. Next, we use the standard linear theory of cosmological perturbations, quantize them, and derive an expression for the symmetrized quantum correlation function of the scalar metric perturbations. This expression is then employed to show the equivalence between this correlation function and that derived from the Einstein-Langevin equation. An alternative proof of this equivalence is provided in Appendix E.

Note that, whereas in stochastic gravity the metric is treated as a classical but stochastic field, in the usual approach to linear cosmological perturbations both metric and inflaton perturbations are quantized. Nevertheless, the correlation functions derived within the Einstein-Langevin approach agree with the symmetrized quantum correlations, similarly to what happens in simpler open quantum systems [33]. More specifically, the stochastic correlation functions derived from the Einstein-Langevin equation agree with the symmetrized quantum correlation functions of the theory of gravity interacting with $N$ matter fields to leading order in $1 / N$ [36]; this was shown in Refs. [37,38] for perturbations around a Minkowski background. In this sense the Einstein-Langevin equation can be regarded as a useful intermediary tool to compute the quantum correlations of metric fluctuations in the large $N$ approximation. It should also be noted that there are situations for open quantum systems where, for a sufficient degree of environment-induced decoherence that guarantees the absence of relevant interference effects, the temporal correlations of some actual properties of the system (corresponding to suitably smeared projectors) can be described in terms of classical stochastic processes governed by a Langevin equation [39]. In those cases the stochastic correlation functions obtained from the Langevin equation also describe such quasiclassical correlations of the system dynamics.

It is, however, important to stress that when one linearizes with respect to both the scalar metric perturbations and the inflaton perturbations, as in the case discussed here, the system cannot be regarded as a true open quantum system. The reason is that Fourier modes decouple and the dynamical constraints due to diffeomorphism invariance link the metric perturbations of scalar type with the perturbations of the inflaton field so that only one true dynamical degree of freedom is left for each Fourier mode.

In Sec. IV we explicitly compute the correlation functions for the scalar metric perturbations in a simple model of chaotic inflation using the Einstein-Langevin approach as described in the previous section. A quasi-de Sitter expansion for the background is assumed and an almost Harrison-Zeldovich spectrum at large scales is obtained. We comment on the different approximations that one is naturally led to consider within the two approaches.

Finally in Sec. V we conclude by summarizing our results and briefly discussing the changes that one would 
encounter when using the Einstein-Langevin equation if the inflaton perturbations were treated exactly, that is, beyond the linear approximation. We note, in particular, that not only scalar but also vectorial and tensorial metric perturbations couple to the inflaton perturbations in that case. The metric perturbations can then be considered a true open quantum system.

Throughout the paper we use the $(+,+,+)$ convention of Ref. [40]. We also make use of the abstract index notation of Ref. [41]. The Latin indices $(a, b, c \ldots)$ denote abstract indices, whereas Greek indices are employed whenever a particular coordinate system is considered [Latin indices such as $(i, j, k \ldots)$ are used instead when referring only to spatial components].

\section{STOCHASTIC GRAVITY FORMALISM AND EINSTEIN-LANGEVIN EQUATION}

\section{A. Stochastic gravity. General formalism}

There are a number of situations, especially in black hole physics and cosmology, in which regarding spacetime as classical whereas the remaining matter fields are quantized has proved very fruitful. This is often considered a reasonable approximation as long as the typical length scales involved are much larger than the Planck length. A first step in that direction is to consider the evolution of quantum matter fields on spacetimes with nonvanishing curvature. The consistent formulation of quantum field theory on general globally hyperbolic spacetimes is nowadays well established for free fields $[42,43]$, and significant progress has been made for interacting fields as well [4446]. Up to this point the quantum matter fields are regarded as test fields evolving on a fixed geometry which is unaffected by their presence.

A second step is to consider the back reaction of quantum matter fields on the spacetime geometry by including the expectation value of the stress tensor operator of the quantum fields as a source of the Einstein equation for the spacetime geometry, which becomes then the so-called semiclassical Einstein equation. The expectation value of the stress tensor is divergent and a nontrivial renormalization procedure is required even for a free field in order to preserve general covariance. This can be achieved by using, for instance, dimensional regularization or point splitting and introducing suitable local counterterms, which are quadratic in the curvature, in the bare gravitational action. Any renormalization method can be used provided Wald's axioms $[42,43]$ are satisfied, since this guarantees equivalent results.

The semiclassical Einstein equation was derived in Ref. [47] by considering the large $N$ limit of $N$ free scalar fields weakly interacting with the gravitational field so that the product of the gravitational coupling constant times the number of fields $N$ remains constant as $N$ tends to infinity; see also Ref. [48] for a related result concerning fermions. However, the semiclassical Einstein equation is most often introduced in an axiomatic way. The basic aspects of this framework, commonly known as semiclassical gravity $[43,49]$, can be summarized as follows. Let us consider a manifold $\mathcal{M}$ with a Lorentzian metric $g_{a b}$ which is globally hyperbolic. Let us also consider a linear matter field evolving on that manifold. In the Heisenberg picture the scalar field operator $\hat{\phi}[g]$ satisfies the Klein-Gordon equation

$$
\left(\nabla_{a} \nabla^{a}-m^{2}\right) \hat{\phi}(x)=0,
$$

where $\nabla_{a}$ means covariant derivative with respect to the metric $g_{a b}$, and the state of the scalar field, which is characterized by a density matrix $\hat{\rho}[g]$, is assumed to be physically acceptable in the sense of Ref. [43]. This means that it is of the so-called Hadamard type, so that the expectation value for the stress tensor operator can be consistently renormalized. The set $\left(\mathcal{M}, g_{a b}, \hat{\phi}[g], \hat{\rho}[g]\right)$ constitutes a self-consistent solution of semiclassical gravity if the following semiclassical Einstein equation is satisfied:

$$
G_{a b}[g]+\Lambda g_{a b}-2\left(\alpha A_{a b}[g]+\beta B_{a b}[g]\right)=\kappa\left\langle\hat{T}_{a b}[g]\right\rangle_{\text {ren }}^{\prime}
$$

where $G_{a b}$ is the Einstein tensor, $\left\langle\hat{T}_{a b}[g]\right\rangle_{\text {ren }}^{\prime}$ is the suitably renormalized expectation value of the stress tensor operator corresponding to the scalar field operator $\hat{\phi}[g]$, and $\alpha$, $\beta, \Lambda$ and $\kappa$ are renormalized parameters (the prime in the expectation value is used to distinguish it from the expectation value introduced below). We considered natural units in which $\hbar=c=1$, and introduced the notation $\kappa=$ $8 \pi G=8 \pi / m_{p}^{2}$ for the renormalized gravitational coupling constant where $m_{p}$ is the Planck mass. The local tensors $A_{a b}$ and $B_{a b}$ are obtained by functionally differentiating with respect to the metric terms in the action that correspond to the Lagrangian densities proportional to $C^{a b c d} C_{a b c d}$ and $R^{2}$, respectively, where $C_{a b c d}$ and $R$ are the Weyl tensor and the scalar curvature. These terms correspond to the finite part of the counterterms introduced in the bare gravitational action to cancel the divergences arising in the expectation value of the stress tensor [42].

From now on, and despite their purely geometric character, we will consider for notational simplicity that the last three terms on the left-hand side of Eq. (2) have been reabsorbed in the renormalized expectation value of the stress tensor operator, which we write now without the prime; this can be done consistently because $\nabla^{a} A_{a b}=0=$ $\nabla^{a} B_{a b}$. Taking this into account, the semiclassical Einstein equation becomes

$$
G_{a b}[g]=\kappa\left\langle\hat{T}_{a b}[g]\right\rangle_{\mathrm{ren}},
$$

where we should now keep in mind that the expectation value depends on the renormalized parameters $\Lambda, \alpha$, and $\beta$. 
There are, however, situations in which the fluctuations of the stress tensor operator are important [50-52]. In Refs. $[53,54]$ it was shown that the semiclassical Einstein equation (2) could be consistently extended to partially account for the fluctuations of the stress tensor operator by introducing a Gaussian stochastic source. More precisely, given a self-consistent solution of semiclassical gravity one can introduce the following equation for the metric perturbations $h_{a b}$ around the background metric $g_{a b}$ :

$$
\begin{aligned}
& G_{a b}[g+h]+\Lambda\left(g_{a b}+h_{a b}\right) \\
& \quad-2\left(\alpha A_{a b}[g+h]+\beta B_{a b}[g+h]\right) \\
& =\kappa\left\langle\hat{T}_{a b}[g+h]\right\rangle_{\text {ren }}^{\prime}+\kappa \xi_{a b}[g],
\end{aligned}
$$

where the whole equation should be understood to linear order in $h_{a b}$. Note that throughout this paper indices will be raised and lowered using the background metric. The renormalized expectation value is computed with the scalar field operator satisfying the Klein-Gordon equation on the perturbed metric $g_{a b}+h_{a b}$ and the Gaussian stochastic source $\xi_{a b}$ is completely determined by the following correlation functions:

$$
\begin{aligned}
\left\langle\xi_{a b}[g ; x)\right\rangle_{\xi}=0, & \\
\left\langle\xi_{a b}[g ; x) \xi_{c d}[g ; y)\right\rangle_{\xi} & =N_{a b c d}(x, y) \\
& =\frac{1}{2}\left\langle\left\{\hat{t}_{a b}[g ; x), \hat{t}_{c d}[g ; y)\right\}\right\rangle,
\end{aligned}
$$

where we used $\langle\ldots\rangle_{\xi}$ to denote the expectation value with respect to the stochastic classical source $\xi_{a b}[g]$. The operator $\hat{t}_{a b}[g]$ is defined as $\hat{t}_{a b}[g] \equiv \hat{T}_{a b}[g]-\left\langle\hat{T}_{a b}[g]\right\rangle$ and the bitensor $N_{a b c d}(x, y)$, which determines the correlation function of the stochastic source, is computed using the scalar field operator satisfying the Klein-Gordon equation for the background metric $g_{a b}$. The bitensor $N_{a b c d}(x, y)$ is called the noise kernel, it describes the quantum fluctuations of the stress tensor operator, and is positivesemidefinite. Strictly speaking, the previous definition for the operator $\hat{t}_{a b}$ only makes sense when some kind of regulator is employed since both the operator $\hat{T}_{a b}[g]$ and the expectation value $\left\langle\hat{T}_{a b}[g]\right\rangle$ are divergent. However, the operator $\hat{t}_{a b}$ is finite in the sense that one can compute any matrix element of this operator using a regularized version of the two terms that define it, and one finally gets a finite result when removing the regulator because the divergences coming from both terms cancel out exactly [54]. Hence, the noise kernel requires no renormalization whereas the divergences of the expectation value $\left\langle\hat{T}_{a b}[g+\right.$ $h]\rangle$ appearing in Eq. (4) are canceled by the counterterms whose finite contribution corresponds to the last three terms on the left-hand side. Furthermore, since $\left\langle\xi_{a b}[g]\right\rangle_{\xi}=0$, Eq. (4), which is called the EinsteinLangevin equation, reduces to the semiclassical Einstein equation for the metric perturbations $h_{a b}$ around the background metric $g_{a b}$ when taking the expectation value with respect to the stochastic source $\xi_{a b}{ }^{2}$ This framework, in which the metric perturbations are regarded as a stochastic process satisfying the Einstein-Langevin equation, is usually referred to as stochastic gravity.

Similarly to what was done for the semiclassical Einstein equation, we will assume that the last three terms on the left-hand side of Eq. (4) are reabsorbed in the renormalized expectation value of the stress tensor operator, so that the Einstein-Langevin equation will be written from now on as

$$
G_{a b}^{(1)}[g+h]=\kappa\left\langle\hat{T}_{a b}^{(1)}[g+h]\right\rangle_{\mathrm{ren}}+\kappa \xi_{a b}[g],
$$

where the superindex (1) means that only terms linear in the metric perturbations $h_{a b}$ are kept. This follows straightforwardly from the fact that Eq. (4) was considered only to linear order in $h_{a b}$ (the stochastic source $\xi_{a b}$ is regarded to be of the same order as $h_{a b}$ ) and that the zeroth-order contribution is identically satisfied, since the background configuration was assumed to be a solution of semiclassical gravity.

A necessary condition for the integrability of the Einstein-Langevin equation, via the Bianchi identity, is the conservation of the stochastic source. Hence, one must make sure that the stochastic source $\xi_{a b}[g]$ is covariantly conserved so that Eq. (7) is a consistent extension of the semiclassical Einstein equation (3). That the stochastic process $\nabla^{a} \xi_{a b}(x)$ vanishes is a consequence of the stress tensor conservation on the background metric [53,54]. Furthermore, it can also be checked that the EinsteinLangevin equation is compatible with the gauge symmetry corresponding to infinitesimal diffeomorphisms. In fact, both the stochastic source and the remaining terms of the Einstein-Langevin equation are separately invariant under gauge transformations for the metric perturbations of the form $h_{a b} \rightarrow h_{a b}+\nabla_{a} \zeta_{b}+\nabla_{b} \zeta_{a}$ corresponding to infinitesimal diffeomorphisms generated by any arbitrary vector field $\vec{\zeta}$ defined on the background spacetime $[53,54]$.

One of the main applications of stochastic gravity is the calculation of the correlation function for the metric perturbations [28,55]. This can be done by solving the Einstein-Langevin equation, which gives a result for the metric perturbation $h_{a b}\left[\xi_{e f} ; x\right)$ in terms of the stochastic source, taking the product $h_{a b}\left[\xi_{e f} ; x\right) h_{c d}\left[\xi_{e f} ; y\right)$ for each solution and averaging over all realizations of the stochastic source (in general averaging over the initial conditions may also be necessary [37]): $\left\langle h_{a b}\left[\xi_{e f} ; x\right) h_{c d}\left[\xi_{e f} ; y\right)\right\rangle_{\xi}$. Note that although the stochastic source and the solutions

\footnotetext{
${ }^{2}$ Remember that Eq. (4) should be understood to linear order in $h_{a b}$. Therefore, when taking the average over all possible realizations of the stochastic source, the equation satisfied by $\left\langle h_{a b}\right\rangle_{\xi}$ coincides with that obtained by linearly perturbing the semiclassical Einstein equation (2).
} 
of the Einstein-Langevin equation are classical stochastic processes, this does not preclude the possibility of obtaining fully quantum correlation functions since the final result for the correlation function only depends on the noise kernel and does not explicitly involve the classical stochastic processes, which can be viewed as an intermediate mathematical tool somewhat analogous to the Feynman histories in a path integral. In fact, it has been exactly shown that a classical stochastic description based on a Langevin equation can be used to obtain the quantum properties of certain kinds of simple open quantum systems [33]. Such a result can be generalized to theories with nonlinear interactions, including the gravitational case. In particular, the quantum correlation functions to leading order in $1 / N$ for gravity interacting with $N$ matter fields coincide with those obtained by solving the EinsteinLangevin equation (this has been shown for perturbations around flat space [37], but can be generalized to arbitrary globally hyperbolic backgrounds [36]).

We finish this general introduction to the EinsteinLangevin equation by briefly mentioning that there are derivations of the Einstein-Langevin equation in different cosmological settings making use of functional methods [56-60] or a derivation using arguments based on the renormalization group [61]. In Appendix $C$ we sketch a derivation of the Einstein-Langevin equation (7) for the case of a general globally hyperbolic background spacetime using the influence functional formalism [54,62]. The Einstein-Langevin equation has also been applied to the study of fluctuations in black hole spacetimes [63-65].

\section{B. Einstein-Langevin equation for cosmological perturbations}

In this paper we will study small perturbations around a Robertson-Walker background when the matter source is a minimally-coupled scalar field with a quadratic potential. In fact, this corresponds to the simplest model of chaotic inflation with the scalar field playing the role of the inflaton field, but it is sufficient for our purpose of illustrating the relationship between the usual treatment of cosmological perturbations and those approaches based on the EinsteinLangevin equation within the framework of stochastic gravity. Furthermore, taking into account the assumptions made throughout the forthcoming sections, the generalization of our main conclusions and results to nonlinear potentials should be rather straightforward as long as we keep to quadratic order in the scalar field perturbations when considering the potential.

Recall that the form for the line element of a general Robertson-Walker metric is

$$
d s^{2}=-d t^{2}+a^{2}(t) \gamma_{i j} d x^{i} d x^{j},
$$

where $a(t)$ is called the scale factor and $\gamma_{i j}$ is the induced metric for the homogeneous spatial sections, which are maximally symmetric hypersurfaces. The line element of the spatial sections can have the three following forms $\gamma_{i j} d x^{i} d x^{j}=\left\{d \chi^{2}+\sin ^{2} \chi d \Omega^{2}, d r^{2}+r^{2} d \Omega^{2}, d \chi^{2}+\right.$ $\left.\sinh ^{2} \chi d \Omega^{2}\right\}$ depending on whether the curvature is positive, zero, or negative, respectively. In terms of the conformal time coordinate $\eta=\int d t a^{-1}(t)$ the metric (8) becomes

$$
d s^{2}=a^{2}(\eta)\left(-d \eta^{2}+\gamma_{i j} d x^{i} d x^{j}\right)
$$

Before proceeding further it is convenient to introduce the following decomposition for the scalar inflaton field, which will be used throughout:

$$
\hat{\phi}(x)=\phi(\eta)+\hat{\varphi}(x),
$$

where $\phi(\eta)$, which corresponds to the expectation value $\langle\hat{\phi}[g ; x)\rangle$ of the inflaton field on the background metric, is a homogeneous classical-like (as an operator it is proportional to the identity) solution of the Klein-Gordon equation which is compatible with the background metric through the semiclassical Einstein equation (3). The operator $\hat{\varphi}(x)$, which will be referred to as the inflaton field perturbations, corresponds to the quantum operator for a minimally-coupled massive scalar field whose expectation value vanishes on the background spacetime, i.e., $\langle\hat{\varphi}[g ; x)\rangle=0$. We will consider a Gaussian state for the inflaton field and, thus, for the inflaton field perturbations; see Appendix A for the definition and the basic properties of pure Gaussian states and the relationship between the state of the inflaton field and the inflaton field perturbations.

It should also be stressed that there are many situations (e.g., in the context of stochastic inflation) in which the classical background configuration of the inflaton field will not be homogeneous over the whole spacetime. Nevertheless, this will not have observable consequences at present provided that the scale of the inhomogeneities is larger than the horizon before the last $60 e$-folds of inflation. In fact, when studying models of eternal inflation [6668] using the formalism of stochastic inflation [69], the expectation value of the inflaton field is no longer the relevant object. One should consider instead the amplitude of a given realization of the inflaton field smeared over scales slightly larger than the horizon radius right before the region that had left the self-regenerating regime and would eventually give rise to our visible Universe underwent the last $60 e$-folds of inflation. It has been argued that in those circumstances the smeared inflaton field behaves as a classical stochastic process. (This is closely related to the quantum to classical transition problem for the inflaton fluctuations [19-26].) If that is the case, one can use a particular realization of the smeared inflaton right before the last $60 e$-folds of inflation as the classical background configuration $\phi(\eta)$ and treat it in the same way in which one would have dealt with a quantum expectation value.

Let us begin by discussing the semiclassical Einstein equation (3) for the background metric $g_{a b}$ defined by 
Eq. (8). The right-hand side of Eq. (3) is the properly renormalized expectation value for the stress tensor of the inflaton field operator, which satisfies the KleinGordon equation (1) on the background spacetime.

If we consider the general expression for the stress tensor operator of a minimally-coupled massive scalar field

$$
\hat{T}_{a b}=\nabla_{a} \hat{\phi} \nabla_{b} \hat{\phi}-\frac{1}{2} g_{a b}\left(g^{c d} \nabla_{c} \hat{\phi} \nabla_{d} \hat{\phi}+m^{2} \hat{\phi}^{2}\right),
$$

and use the decomposition of the scalar field introduced in Eq. (10), the expectation value for the stress tensor operator can be separated into three different contributions:

$$
\left\langle\hat{T}_{a b}[g]\right\rangle_{\mathrm{ren}}=\left\langle\hat{T}_{a b}[g]\right\rangle_{\phi \phi}+\left\langle\hat{T}_{a b}[g]\right\rangle_{\phi \varphi}+\left\langle\hat{T}_{a b}[g]\right\rangle_{\varphi \varphi}^{\mathrm{ren}},
$$

where the subindices $\phi \phi, \phi \varphi$ and $\varphi \varphi$ are used to denote the contributions to the stress tensor operator which are, respectively, quadratic in $\phi(\eta)$, linear in both $\phi(\eta)$ and $\hat{\varphi}(x)$, and quadratic in $\hat{\varphi}(x)$. The first term depends just on the homogeneous solution $\phi(\eta)$, the second term vanishes since it is proportional to $\langle\hat{\varphi}[g ; x)\rangle$, and the third term, which is completely independent of the homogeneous part $\phi(\eta)$, is quadratic in the inflaton field perturbations $\hat{\varphi}(x)$ and needs renormalization.

The first term on the right-hand side of Eq. (12) will be denoted by $\mathcal{T}_{a b} \equiv\left\langle\hat{T}_{a b}[g]\right\rangle_{\phi \phi}$; see Appendix B for further comments on this notation. Taking into account the special form of the Robertson-Walker metric, in the basis associated with the conformal time and comoving spatial coordinates these components can be rewritten as

$$
\begin{gathered}
\mathcal{T}_{00}=\frac{1}{2}\left(\left(\phi^{\prime}\right)^{2}+m^{2} a^{2} \phi^{2}\right), \\
\mathcal{T}_{i j}=\frac{1}{2}\left(\left(\phi^{\prime}\right)^{2}-m^{2} a^{2} \phi^{2}\right) \gamma_{i j},
\end{gathered}
$$

where primes denote derivatives with respect to the conformal time $\eta$. In this coordinate system the time-time and space-space components of $a^{-2}(\eta) \mathcal{T}_{\mu \nu}$ can be, respectively, identified with the energy density $\rho(\eta)$ and the isotropic pressure $p(\eta)$ of a perfect fluid. The components of Eq. (3) become then the usual Friedmann equations

$$
\begin{gathered}
\frac{\kappa}{2}\left(\left(\phi^{\prime}\right)^{2}+m^{2} a^{2} \phi^{2}\right)=3\left(\mathcal{H}^{2}+\epsilon\right), \\
\frac{\kappa}{2}\left(\left(\phi^{\prime}\right)^{2}-m^{2} a^{2} \phi^{2}\right)=-\left(2 \mathcal{H}^{\prime}+\mathcal{H}^{2}+\epsilon\right),
\end{gathered}
$$

where $\mathcal{H}=a^{\prime} / a$ and $\epsilon=0,1,-1$ depending on whether the homogeneous spatial sections of the Robertson-Walker geometry are, respectively, flat, with positive curvature or with negative curvature.

The third term on the right-hand side of Eq. (12), $\left\langle\hat{T}_{a b}[g]\right\rangle_{\varphi \varphi}^{\text {ren }}$, will in turn have a similar structure to that of
Eqs. (13) and (14) with diagonal nonvanishing components which can be regarded as corrections $\Delta \rho(\eta)$ and $\Delta p(\eta)$ to the energy density and pressure. This structure is necessary so that the solutions of Eq. (3) are of Robertson-Walker type, but there is a family of quantum states of the scalar field which gives rise to such a structure for $\left\langle\hat{T}_{a b}[g]\right\rangle_{\varphi \varphi}^{\text {ren }}$. They can be characterized as follows. Since the Lie derivatives of the six spacelike Killing vectors which characterize a Robertson-Walker metric commute with the Klein-Gordon operator satisfying Eq. (1), one can introduce a unitary operator which implements at the quantum level the symmetries corresponding to the six Killing vectors and is preserved by the dynamical evolution. Consequently, the Hadamard function (the quantum expectation value of the anticommutator of the field) employed to compute the renormalized expectation value of the field $\hat{\varphi}(x)$ will respect the symmetries of the Robertson-Walker geometry provided that one considers a quantum initial state which is kept invariant, up to a phase, by the unitary operator associated with those symmetries. Throughout this paper we will consider this class of states (spatially homogeneous and isotropic). Nevertheless, being quadratic in the inflaton perturbations, which are considered in general to be much smaller during the inflationary period than the homogeneous background solution $\phi(\eta)$, the contribution from the last term in Eq. (12) and, hence, the corrections $\Delta \rho$ and $\Delta p$, will in general be small compared to those from Eqs. (13) and (14) during the inflationary period. The usual treatments which keep to linear order in both the metric perturbations and the inflaton perturbations directly discard them. This is actually the situation that we will be interested in here. Therefore, the background solution for the scale factor $a(\eta)$ is completely determined by Eqs. (15) and (16) without considering the corrections that come from the third term on the right-hand side of Eq. (12), which is approximated to linear order by $\left\langle\hat{T}_{a b}[g]\right\rangle_{\mathrm{ren}} \approx \mathcal{T}_{a b}$.

In addition, either from the conservation of the stress tensor $\nabla^{a} \mathcal{T}_{a b}=0$ or by taking the expectation value of Eq. (1), the homogeneous background solution $\phi(\eta)$ is seen to satisfy the following Klein-Gordon equation on the background Robertson-Walker metric:

$$
\phi^{\prime \prime}+2 \frac{a^{\prime}}{a} \phi^{\prime}+m^{2} a^{2} \phi=0
$$

Let us now consider the objects which appear in the Einstein-Langevin equation (7) and particularize them to the case addressed here. The geometric part, i.e., the components of the Einstein tensor for a linear perturbation $h_{a b}$ of the metric will be discussed in the next section. The contribution to the expectation value of the stress tensor which is linear in the metric perturbation, $\left\langle\hat{T}_{a b}^{(1)}[g+h]\right\rangle_{\text {ren }}$, can be decomposed according to Eq. (10) as 


$$
\begin{aligned}
\left\langle\hat{T}_{a b}^{(1)}[g+h]\right\rangle_{\mathrm{ren}}= & \left\langle\hat{T}_{a b}^{(1)}[g+h]\right\rangle_{\phi \phi}+\left\langle\hat{T}_{a b}^{(1)}[g+h]\right\rangle_{\phi \varphi} \\
& +\left\langle\hat{T}_{a b}^{(1)}[g+h]\right\rangle_{\varphi \varphi}^{\text {ren }},
\end{aligned}
$$

where the whole inflaton field satisfies now the KleinGordon equation on the perturbed metric $\tilde{g}_{a b}=g_{a b}+h_{a b}$,

$$
\left(\tilde{\nabla}_{a} \tilde{\nabla}^{a}-m^{2}\right) \hat{\phi}=0,
$$

and $\tilde{\nabla}_{a}$ means the covariant derivative with respect to $\tilde{g}_{a b}$. The first term on the right-hand side of Eq. (18), $\left\langle\hat{T}_{a b}^{(1)}\right\rangle_{\phi \phi}$, depends on the scalar field only via the homogeneous background solution $\phi(\eta)$, which was already fixed by Eq. (17) together with Eqs. (15) and (16), and therefore the metric perturbations enter only through the explicit dependence of the stress tensor on the metric. Contrary to what happened in Eq. (12), the second term on the righthand side of Eq. (18) $\left\langle\hat{T}_{a b}^{(1)}\right\rangle_{\phi \varphi}$ no longer vanishes since it is now proportional to $\langle\hat{\varphi}[g+h]\rangle$ and the Klein-Gordon equation satisfied by $\hat{\varphi}[g+h]$ on the spacetime with the perturbed metric $\tilde{g}_{a b}$, given by Eq. (19), has an inhomogeneous source term proportional to the metric perturbation $h_{a b}$ and the homogeneous background solution $\phi(\eta)$ which in general prevents its expectation value $\langle\hat{\varphi}[g+$ $h]\rangle$ from vanishing. Hence, the only nonvanishing contributions to the second term are those which depend implicitly on the metric perturbations through the quantum operator for the inflaton perturbations $\hat{\varphi}[g+h]$.

Finally, the third term $\left\langle\hat{T}_{a b}^{(1)}\right\rangle_{\varphi \varphi}^{\text {ren }}$ which requires renormalization will have contributions with either explicit or implicit dependence on the metric perturbation. Not only the contributions which depend on the metric perturbations explicitly but also those which depend implicitly via $\hat{\varphi}[g+h]$ are ultimately quadratic in the inflaton perturbations $\hat{\varphi}[g]$ [after solving Eq. (19) perturbatively in the metric perturbations]; otherwise they would vanish, as follows from the fact that $\langle\hat{\varphi}[g]\rangle=0$.

Similarly to what was said concerning the last term in Eq. (12), the last term in Eq. (18) is not taken into account by usual approaches to cosmological perturbations, which keep to linear order in the inflaton perturbations as well as the metric perturbations. We will not consider these terms either in the next two sections, but some general remarks on how to deal with them, and possible implications, will be made in Sec. V.

Let us now briefly concentrate on the noise kernel, which accounts for the stress tensor fluctuations and characterizes the correlations of the stochastic source $\xi_{a b}$. It is proportional to $\left\langle\left\{\hat{t}_{a b}[g], \hat{t}_{c d}[g]\right\}\right\rangle$ where $\hat{t}_{a b}=\hat{T}_{a b}-\left\langle\hat{T}_{a b}\right\rangle$, and is evaluated on the background metric. Using Eqs. (10) and (11) it can be separated into the following nonvanishing terms:

$$
\begin{aligned}
\left\langle\left\{\hat{t}_{a b}[g], \hat{t}_{c d}[g]\right\}\right\rangle= & \left\langle\left\{\hat{t}_{a b}[g], \hat{t}_{c d}[g]\right\}\right\rangle_{\phi \varphi} \\
& +\left\langle\left\{\hat{t}_{a b}[g], \hat{t}_{c d}[g]\right\}\right\rangle_{\varphi \varphi},
\end{aligned}
$$

where the first and second terms on the right-hand side are, respectively, quadratic and quartic in the inflaton perturbation $\hat{\varphi}[g]$. We used a notation similar to that introduced in Eq. (12) since the first term on the right-hand side of Eq. (20) comes entirely from those contributions to the operators $\hat{t}_{a b}$ and $\hat{t}_{c d}$ which are proportional to both $\phi(\eta)$ and $\hat{\varphi}[g]$, whereas the last term in Eq. (20) comes from the contributions to the stress tensor which are quadratic in $\hat{\varphi}[g]$. The contribution to the noise kernel which depends on the background homogeneous solution $\phi(\eta)$ but not on the inflaton perturbation $\hat{\varphi}$ vanishes since, being proportional to the identity, the corresponding stress tensor operator coincides with its expectation value. The terms linear in $\hat{\varphi}[g]$ also vanish because $\langle\hat{\varphi}[g]\rangle=0$. Finally, since we will be considering Gaussian quantum states for the inflaton perturbations (see Appendix A for a definition and a brief description of some basic properties of Gaussian states), the 3-point quantum correlation functions $\langle\hat{\varphi}[g] \hat{\varphi}[g] \hat{\varphi}[g]\rangle$ are proportional to the expectation value $\langle\hat{\varphi}[g]\rangle$ and, therefore, the contributions which are cubic in $\hat{\varphi}[g]$ vanish as well.

It is important to note that both the quadratic and the quartic contributions to the noise kernel are separately conserved since both $\phi(\eta)$ and $\hat{\varphi}[g ; x)$ independently satisfy the Klein-Gordon equation (10) on the background geometry; recall that $\phi(\eta)=\langle\hat{\phi}[g]\rangle$. Because of this fact, we can consistently consider a pair of independent stochastic sources $\xi_{1 a b}$ and $\xi_{2 a b}$ associated with each term so that $\xi_{a b}=\xi_{1 a b}+\xi_{2 a b} \quad$ with $\quad\left\langle\xi_{1 a b}(x) \xi_{1 c d}\left(x^{\prime}\right)\right\rangle=\left\langle\left\{\hat{t}_{a b}(x)\right.\right.$, $\left.\left.\hat{t}_{c d}\left(x^{\prime}\right)\right\}\right\rangle_{\phi \varphi}[g],\left\langle\xi_{2 a b}(x) \xi_{2 c d}\left(x^{\prime}\right)\right\rangle=\left\langle\left\{\hat{t}_{a b}(x), \hat{t}_{c d}\left(x^{\prime}\right)\right\}\right\rangle_{\varphi \varphi}[g]$, and $\left\langle\xi_{1 a b}(x) \xi_{2 c d}\left(x^{\prime}\right)\right\rangle=0$. The integrability of the Einstein-Langevin equation with any of the two sources is then guaranteed because both sources are separately conserved.

In the next section we will show that keeping only $\xi_{1 a b}$, which can be thought to be of the same order as $\hat{\varphi}$, the results obtained using the Einstein-Langevin equation and those from the usual treatments which quantize the linearized theory for both the metric and the inflaton perturbations are equivalent. On the other hand, some of the main features and consequences of the source $\xi_{2 a b}$, which can be regarded as being of quadratic order in $\hat{\varphi}$, will be briefly discussed in Sec. V and studied in more detail in Ref. [36]. Of course, when considering the stochastic source $\xi_{2 a b}$, the last term on the right-hand side of Eqs. (12) and (18) should also be considered since their contribution is of the same order as that of $\xi_{2 a b}$.

\section{EINSTEIN-LANGEVIN EQUATION FOR LINEARIZED COSMOLOGICAL PERTURBATIONS}

\section{A. Gauge-invariant formalism for linearized cosmological perturbations}

Let us consider small metric perturbations around a fixed Robertson-Walker background geometry. It can be shown 
[70] that the most general expression for the components of metric perturbations in some particular coordinate system can be written as

$$
\begin{gathered}
h_{00}=2 \bar{\phi} a^{2}(\eta), \quad h_{0 i}=-\left(B_{\mid i}+S_{i}\right) a^{2}(\eta), \\
h_{i j}=\left[2\left(\psi \gamma_{i j}-E_{\mid i j}\right)+\left(F_{i \mid j}+F_{j \mid i}\right)+h_{i j}\right] a^{2}(\eta),
\end{gathered}
$$

which depends on ten functions: $\bar{\phi}, \psi, B$, and $E$, the two independent components of each transverse vector $S_{i}$ and $F_{i}$, and the two independent components of the traceless and transverse symmetric tensor $h_{i j}$. The vectors $S_{i}$ and $F_{i}$ as well as the tensor $h_{i j}$ are tangent to the isotropic and homogeneous spatial sections of the Robertson-Walker spacetime, but depend in general on the conformal time $\eta$ which labels each spatial section. Furthermore, the notation $\mid i$ is used to denote the covariant derivative associated with the metric $\gamma_{i j}$ induced on these spatial sections. The transversality condition for the vectors and tensor is then written as $S_{\mid i}^{i}=0, F_{\mid i}^{i}=0$, and $h_{j \mid i}^{i}=0$. The global factor $a^{2}(\eta)$ is introduced for later convenience, but could be reabsorbed. The metric perturbations will henceforth be treated linearly.

Four functions describing the metric perturbations are of scalar type, four more are of vector type, and finally there are two which are of tensor type, according to their transformation properties on the three-dimensional spatial sections [70-72]. These types are preserved by time evolution provided that the perturbations of the matter sources around the configuration generating the background Robertson-Walker geometry are also treated linearly. Those ten functions do not characterize in a unique way nonequivalent perturbed geometries since they may arise not only due to real perturbations of the geometry but also to changes of the mapping from the background manifold to the perturbed one. Hence, a diffeomorphism generated by a vector field $\vec{\zeta}$, considered to be of the same order as the metric perturbations, would give an extra contribution $\mathcal{L}_{\dot{\xi}} g_{a b}$ to the metric perturbation $h_{a b}$, where $g_{a b}$ is the background metric. These local diffeomorphisms do not preserve in general the scalar, vectorial, or tensorial nature of the metric perturbations.

There are different approaches to overcome the difficulties derived from this gauge freedom. One approach is to fix the gauge [73] so that further changes on the metric perturbations resulting from coordinate changes are not allowed. This can be achieved by fixing some of the ten functions characterizing the components of the metric perturbations either directly specifying some components of $h_{a b}$ or imposing relations between them. A second approach, first used by Bardeen [71], is based on the introduction of so-called gauge-invariant variables, which corresponds to using linear combinations of those ten functions which remain invariant to linear order under diffeomorphisms generated by any vector field $\vec{\zeta}$. One can always argue that those gauge-invariant variables co- incide with the value taken by the functions appearing in Eq. (21) (or some linear combination of them) in some particular gauge, as follows from the remark that the components of any tensorial object referred to a particular and fixed coordinate system do not change when reexpressed in terms of some new coordinates [74].

From now on we will consider spatially-flat RobertsonWalker metrics, i.e. $\gamma_{i j}=\delta_{i j}$ in Eq. (8), and concentrate on scalar-type metric perturbations. The motivation for the latter is that scalar-type metric perturbations are the only ones which couple to matter sources characterized by scalar functions when both metric perturbations and matter perturbations (the inflaton perturbations in our case) are treated linearly. Only two true kinematical degrees of freedom (i.e., before imposing the Einstein equation) exist for this type of perturbation, in the sense that from the four arbitrary functions characterizing scalar metric perturbations, the equivalence classes invariant under local diffeomorphism transformations are completely characterized by two arbitrary functions [70,75-77]. A particular example corresponds to the following two linear combinations of the four functions $\bar{\phi}, \psi, B$, and $E$, which are invariant under local diffeomorphisms:

$$
\Phi=\bar{\phi}+\frac{1}{a}\left[\left(B-E^{\prime}\right) a\right]^{\prime}, \quad \Psi=\psi-\frac{a^{\prime}}{a}\left(B-E^{\prime}\right) .
$$

These gauge-invariant variables were first introduced by Bardeen [71] with the notation $\Phi_{A}=\Phi$ and $\Phi_{H}=-\Psi$.

One can also define a gauge-invariant version of the linear perturbations of the Einstein tensor, $\left(G_{\text {inv }}^{(1)}\right)_{a}^{b}$, which depends only on the gauge-invariant functions $\Phi$ and $\Psi$, and is invariant under the same kind of local diffeomorphism which preserves the scalar nature of the metric perturbations characterized by $\Phi$ and $\Psi$; see Ref. [76,77] for details. In fact, the gauge-invariant perturbations $\left(G_{\text {inv }}^{(1)}\right)_{a}^{b}$ of the Einstein tensor coincide with the actual components of the linear perturbations of the Einstein tensor in the so-called longitudinal gauge, which corresponds to taking $E=B=0$. Similarly, one could also define a gauge-invariant version of the stress tensor linear perturbations and write a version of the Einstein equation for the metric perturbations with both sides explicitly invariant; recall that the whole linearized Einstein equation is itself gauge invariant. We will follow an alternative procedure which yields equivalent results. The idea is to consider the components of the Einstein equation in the longitudinal gauge and notice, as will be explicitly shown below, that all the geometric dependence can be written entirely in terms of the gauge-invariant variables $\Phi$ and $\Psi$ since the only nonvanishing scalar contributions to the metric perturbations in the longitudinal gauge, $\bar{\phi}$ and $\psi$, coincide with $\Phi$ and $\Psi$.

In the longitudinal gauge the expression of the perturbed metric for scalar-type perturbations on a spatially-flat Robertson-Walker background in terms of the two gauge- 
invariant functions $\Phi(x)$ and $\Psi(x)$ is

$d s^{2}=a^{2}(\eta)\left[-(1+2 \Phi(x)) d \eta^{2}+(1-2 \Psi(x)) \delta_{i j} d x^{i} d x^{j}\right]$,

and the components of the linear perturbation of the Einstein tensor are

$$
\begin{gathered}
G_{0}^{(1) 0}=2 a^{-2}\left[3 \mathcal{H}\left(\mathcal{H} \Phi+\Psi^{\prime}\right)-\nabla^{2} \Psi\right] \\
G^{(1) i}{ }_{0}=2 a^{-2} \partial_{i}\left(\mathcal{H} \Phi+\Psi^{\prime}\right), \\
G_{i}^{(1) j}=2 a^{-2}\left[\left(2 \mathcal{H}^{\prime}+\mathcal{H}^{2}\right) \Phi+\mathcal{H} \Phi^{\prime}+\Psi^{\prime \prime}+2 \mathcal{H} \Psi^{\prime}\right. \\
\left.+\frac{1}{2} \nabla^{2} D\right] \delta_{i}^{j}-a^{-2} \delta^{j k} \partial_{k} \partial_{i} D
\end{gathered}
$$

where $\mathcal{H}=a^{\prime}(\eta) / a(\eta), D=\Phi-\Psi, \nabla^{2}=\delta^{i j} \partial_{i} \partial_{i}$ and, as we mentioned above, primes denote derivation with respect to the conformal time $\eta$. The Einstein equation for the linear perturbations of the metric is

$$
G_{a}^{(1) b}=\kappa\left(T_{a}^{(0) b}-\left\langle T_{a}^{(0) b}\right\rangle+T_{a}^{(1) b}\right),
$$

where the right-hand side corresponds to those terms which are not included in the background stress tensor $\mathcal{T}_{a}^{b}$ and are at most linear in the metric perturbations (some terms from the stress tensor on the background geometry $T^{(0) b}{ }_{a}$ are present because the scalar field is also perturbed). Note that at the classical level one should simply substitute the unperturbed stress tensor $\mathcal{T}_{a}^{b}$ for $\left\langle T^{(0) b}{ }_{a}\right\rangle$. However, $\left\langle T^{(0) b}{ }_{a}\right\rangle$ has nontrivial additional components in stochastic gravity or when quantizing both the metric perturbations and the scalar field; see the last paragraph of Appendix B for additional discussion on the notation and related points. Furthermore, from now on we will only consider terms which are linear in either the metric perturbations or the inflaton field perturbations $\varphi$, which are both assumed to be of the same order. The contribution to the stress tensor of these linear perturbations will be denoted by $\delta \mathcal{T}_{a}^{b}$. Hence, taking all this into account, the components of the Einstein equation for linear scalar perturbations of the metric become

$$
G^{(1) b}{ }_{a}=\kappa \delta \mathcal{T}_{a}^{b} .
$$

Let us remember that the expression for the stress tensor of the free massive field $\varphi$, which is minimally coupled to the spacetime curvature and evolves on the perturbed metric $\tilde{g}_{a b}=g_{a b}+h_{a b}$, is

$$
T_{a b}=\tilde{\nabla}_{a} \varphi \tilde{\nabla}_{b} \varphi-\frac{1}{2} \tilde{g}_{a b}\left(\tilde{g}^{c d} \tilde{\nabla}_{c} \varphi \tilde{\nabla}_{d} \varphi+m^{2} \varphi^{2}\right) .
$$

The components for the linear perturbations of the stress tensor, $\delta \mathcal{T}_{a}^{b}$ in the basis associated with the conformal time and comoving spatial coordinates are then straightforwardly obtained:

$$
\begin{gathered}
\delta \mathcal{T}_{0}^{0}=a^{-2}\left(\Phi\left(\phi^{\prime}\right)^{2}-\phi^{\prime} \varphi^{\prime}-m^{2} a^{2} \phi \varphi\right), \\
\delta \mathcal{T}_{0}^{i}=a^{-2} \phi^{\prime} \partial_{i} \varphi, \\
\delta \mathcal{T}_{i}^{j}=a^{-2} \delta_{i}^{j}\left(-\Phi\left(\phi^{\prime}\right)^{2}+\phi^{\prime} \varphi^{\prime}-m^{2} a^{2} \phi \varphi\right) .
\end{gathered}
$$

Taking into account the fact that $\delta \mathcal{T}_{i}^{j}$ is diagonal, one can use Eq. (27) [see also Eq. (25)] with $i \neq j$ to conclude that $\Phi$ and $\Psi$ are equal [76,77], except for a possible homogeneous component (independent of the spatial coordinates), which should be included in the background scale factor. Alternatively, the same conclusion can also be reached by considering the sum of all the diagonal elements of the $i j$-components of Eq. (27) together with Eqs. (30) and (31) to substitute $\varphi$ in terms of $\Phi$ and $\Psi$, which yields $\nabla^{2}(\Phi-$ $\Psi)=0$ and hence $\Psi=\Phi$ provided that they vanish at infinity.

From the Friedmann equations (15) and (16) for the background solution, we have

$$
\frac{\kappa}{2}\left(\phi^{\prime}\right)^{2}=\mathcal{H}^{2}-\mathcal{H}^{\prime},
$$

and we can use this equation to reexpress the terms in Eqs. (29)-(31) which are linear in $\Phi$. Substituting these terms into the perturbed Einstein equations, given by Eq. (27), we finally get

$$
\begin{gathered}
\nabla^{2} \Phi-3 \mathcal{H} \Phi^{\prime}-\left(\mathcal{H}^{\prime}+2 \mathcal{H}^{2}\right) \Phi \\
=\frac{\kappa}{2}\left(\phi^{\prime} \varphi^{\prime}+m^{2} a^{2} \phi \varphi\right) \\
\partial_{i}\left(\Phi^{\prime}+\mathcal{H} \Phi\right)=\frac{\kappa}{2} \phi^{\prime} \partial_{i} \varphi
\end{gathered}
$$

$\left(\mathcal{H}^{\prime}+2 \mathcal{H}^{2}\right) \Phi+3 \mathcal{H} \Phi^{\prime}+\Phi^{\prime \prime}=\frac{\kappa}{2}\left(\phi^{\prime} \varphi^{\prime}-m^{2} a^{2} \phi \varphi\right)$

Similarly, the Klein-Gordon equation for the inflaton perturbations can be obtained by linearizing in both the metric perturbations $\Phi$ and the inflaton perturbations $\varphi$ the exact Klein-Gordon equation for the whole inflaton field $\phi(\eta)+\varphi(x)$ on the perturbed geometry, Eq. (19), and making use of the fact that the homogeneous background solution $\phi(\eta)$ satisfies the Klein-Gordon equation on the background spacetime, Eq. (17). It can also be obtained from the conservation equation, to linear order in the metric perturbations, of the linearly perturbed stress tensor. The result is

$$
\varphi^{\prime \prime}+2 \mathcal{H} \varphi^{\prime}-\nabla^{2} \varphi+m^{2} a^{2} \varphi-4 \phi^{\prime} \Phi^{\prime}+2 m^{2} a^{2} \phi \Phi=0,
$$

where we have already taken into account that $\Psi=\Phi$. 


\section{B. Einstein-Langevin equation for linearized cosmological perturbations}

Using the results of the previous subsection, the Einstein-Langevin equation (7) can be particularized to the case of scalar-type metric perturbations around a Robertson-Walker background geometry with the following result:

$$
\begin{aligned}
\frac{\kappa}{2} a^{2}\left(\left\langle\delta \hat{\mathcal{T}}_{0}^{0}\right\rangle_{\Phi}+\xi_{0}^{0}\right) & =3 \mathcal{H}\left(\mathcal{H} \Phi+\Psi^{\prime}\right)-\nabla^{2} \Psi \\
\frac{\kappa}{2} a^{2}\left(\left\langle\delta \hat{\mathcal{T}}_{0}^{i}\right\rangle_{\Phi}\right. & \left.+\xi_{0}^{i}\right)=\partial_{i}\left(\Psi^{\prime}+\mathcal{H} \Phi\right), \\
\frac{\kappa}{2} a^{2}\left(\left\langle\delta \hat{\mathcal{T}}_{i}^{j}\right\rangle_{\Phi}+\xi_{i}^{j}\right)= & {\left[\left(2 \mathcal{H}^{\prime}+\mathcal{H}^{2}\right) \Phi+\mathcal{H} \Phi^{\prime}+\Psi^{\prime \prime}\right.} \\
& \left.+2 \mathcal{H} \Psi^{\prime}+\frac{1}{2} \nabla^{2} D\right] \delta_{i}^{j} \\
& -\frac{1}{2} \delta^{j k} \partial_{k} \partial_{i} D,
\end{aligned}
$$

where we have used Eqs. (23)-(25) for the linearized Einstein tensor and we have considered the stress tensor operator $\delta \hat{\mathcal{T}}_{a}^{b}$, which results from keeping terms linear in either the inflaton perturbations or the metric perturbations. The notation $\left\langle\delta \hat{\mathcal{T}}_{a}^{b}\right\rangle_{\Phi}$ is equivalent to $\left\langle\delta \hat{\mathcal{T}}_{a}^{b}[g+h]\right\rangle$ for the particular case of scalar metric perturbations that we are considering. Note, in addition, that all the contributions to $\left\langle\delta \hat{\mathcal{T}}_{a}^{b}\right\rangle_{\Phi}$ are, explicitly or implicitly, proportional to the metric perturbations since otherwise they would be proportional to $\langle\hat{\varphi}[g]\rangle$, which vanishes. In fact, this turns out to be important so that $\left\langle\delta \hat{\mathcal{T}}_{a}^{b}[g+h]\right\rangle$ coincides with $\left\langle\hat{T}^{(1) b}{ }_{a}[g+h]\right\rangle$, which is the object that appears in the Einstein-Langevin equation, when one keeps to linear order in the inflaton perturbations, i.e., when only the first two terms on the right-hand side of Eq. (18) are considered.

The three equations corresponding to the spatial components with equal indices of the Einstein-Langevin equation are equivalent due to the symmetries of the RobertsonWalker metric and those of the Gaussian state of the inflaton perturbations being considered, which was chosen to be compatible with those symmetries. On the other hand, the equation for the spatial components with different indices can be used in a similar way to that of the previous subsection in order to show that the gauge-invariant functions for the scalar metric perturbations $\Phi(x)$ and $\Psi(x)$ coincide. In this case it is also necessary that the spatial components of the stochastic source $\xi_{i j}(x)$ with indices $i \neq$ $j$ vanish identically. Indeed, since $\xi_{a b}(x)$ is a Gaussian stochastic process with zero mean, $\xi_{i j}(x)$ will vanish provided that $\left\langle\xi_{i j}(x) \xi_{c d}(y)\right\rangle_{\xi}=0$, which can be argued as follows. The correlation function for $\xi_{a b}$ is defined by the noise kernel and, as we are keeping to linear order in the inflaton perturbations, only the first contribution in Eq. (20), $\left\langle\left\{\hat{t}_{i j}[g], \hat{t}_{c d}[g]\right\}\right\rangle_{\phi \varphi}=\left\langle\left\{\delta \hat{t}_{i j}[g], \delta \hat{t}_{c d}[g]\right\}\right\rangle$, where $\delta \hat{t}_{a b} \equiv \delta \hat{\mathcal{T}}_{a b}-\left\langle\delta \hat{\mathcal{T}}_{a b}\right\rangle$, should be considered. Finally, $\left\langle\left\{\delta \hat{t}_{i j}[g], \delta \hat{t}_{c d}[g]\right\}\right\rangle$ vanishes for $i \neq j$ since $\delta \hat{\mathcal{T}}_{i j}=0$ in that case, as follows from Eq. (31) with the inflaton perturbation promoted to a quantum operator. Hence, from now on we will take $\Psi=\Phi$. Equation (39) is then trivially satisfied for $i \neq j$ and for $i=j$ it reduces to (no summation should be understood over the repeated index $i$ )

$$
\frac{\kappa}{2} a^{2}\left(\left\langle\delta \hat{\mathcal{T}}_{i}^{i}\right\rangle_{\Phi}+\xi_{i}^{i}\right)=\left(2 \mathcal{H}^{\prime}+\mathcal{H}^{2}\right) \Phi+3 \mathcal{H} \Phi^{\prime}+\Phi^{\prime \prime}
$$

It is clear that Eqs. (37)-(39) are redundant since we have three equations but only two variables to be determined: the function $\Phi$ characterizing the metric perturbations of scalar type and the expectation value of the quantum operator for the inflaton perturbations on the spacetime with the perturbed metric, $\langle\hat{\varphi}[g+h]\rangle$, which will also be denoted in this case by $\langle\hat{\varphi}\rangle_{\Phi}$. However, despite the apparently excessive number of equations, the system is integrable and solutions can be found. This fact is guaranteed by the Bianchi identity provided that the source of the Einstein-Langevin equation is conserved. This is indeed the case: the averaged and stochastic sources are separately conserved. On the one hand, the conservation of $\left\langle\delta \hat{\mathcal{T}}_{a b}\right\rangle_{\Phi}$ is equivalent to the Klein-Gordon equation for the expectation value $\langle\hat{\varphi}\rangle_{\Phi}$, which is completely analogous to Eq. (36):

$$
\begin{array}{r}
\langle\hat{\varphi}\rangle_{\Phi}^{\prime \prime}+2 \mathcal{H}\langle\hat{\varphi}\rangle_{\Phi}^{\prime}-\nabla^{2}\langle\hat{\varphi}\rangle_{\Phi}+m^{2} a^{2}\langle\hat{\varphi}\rangle_{\Phi}-4 \phi^{\prime} \Phi^{\prime} \\
+2 m^{2} a^{2} \phi \Phi=0 .
\end{array}
$$

On the other hand, the conservation of the stochastic source is a consequence of the conservation of the noise kernel, which in turn relies on the fact that the quantum operator for the inflaton perturbations $\hat{\varphi}[g]$ satisfies the KleinGordon equation on the background spacetime $\left(\nabla_{a} \nabla^{a}-\right.$ $\left.m^{2}\right) \hat{\varphi}(x)=0$.

Taking all these considerations into account, the KleinGordon equation (41) can be used to obtain the expectation value $\langle\hat{\varphi}\rangle_{\Phi}$ in terms of $\Phi$. We can then easily write the expectation value of the stress tensor linear perturbations $\left\langle\delta \hat{\mathcal{T}}_{a}^{b}\right\rangle_{\Phi}$ in terms of $\Phi$ and use any of the constraint equations, Eq. (37) or (38), to express $\Phi$ entirely in terms of the stochastic source $\xi_{a b}$; to be specific, in this subsection we will consider Eq. (38). The spatial derivatives can be easily handled by working in Fourier space. Hence, in the rest of this section we will work with Fouriertransformed expressions in the spatial coordinates. A subindex $k$ will denote the three-dimensional comoving momentum vector $\vec{k}$ that labels each Fourier mode in flat space, i.e.,

$$
\Phi_{k}(\eta)=\int d^{3} x e^{-i \vec{k} \cdot \vec{x}} \Phi(\eta, \vec{x}) .
$$

Thus, the Fourier-transformed version of Eq. (38) is 


$$
i k_{i}\left(\Phi_{k}^{\prime}+\mathcal{H} \Phi_{k}\right)=\frac{\kappa}{2} a^{2}\left(\left\langle\left(\delta \hat{\mathcal{T}}_{0}^{i}\right)_{k}\right\rangle_{\Phi}+\left(\xi_{0}^{i}\right)_{k}\right),
$$

where $k_{i}$ is the comoving momentum component associated with the comoving coordinate $x^{i}$.

Since $\left\langle\delta \hat{\mathcal{T}}_{a}^{b}\right\rangle_{\Phi}$ is linear in $\Phi$, Eq. (43) is a first-order linear integro-differential equation with an inhomogeneous term corresponding to the $0 i$ component of the stochastic source $\xi_{a}^{b}$. Therefore, one can always write the solution to Eq. (43) for $\eta \geq \eta_{0}$ as

$$
\begin{aligned}
\Phi_{k}(\eta) & =\Phi_{k}^{(\mathrm{h})}(\eta)+\Phi_{k}^{(\mathrm{i})}(\eta) \\
& =\Phi_{k}^{(\mathrm{h})}(\eta)+\frac{\kappa}{2} \int_{\eta_{0}}^{\eta} d \eta^{\prime} G_{\mathrm{ret}}^{(k)}\left(\eta, \eta^{\prime}\right) a^{2}\left(\eta^{\prime}\right)\left(\xi_{0}^{i}\right)_{k}\left(\eta^{\prime}\right),
\end{aligned}
$$

where $\Phi_{k}^{(\mathrm{h})}$ is the solution to the homogeneous version of Eq. (43) with some given initial conditions at an initial time $\eta_{0}, \Phi_{k}^{(i)}$ is a solution of the inhomogeneous equation which vanishes at $\eta_{0}$, and $G_{\text {ret }}^{(k)}$ is the retarded propagator associ- ated with Eq. (43). The correlation function for the scalar metric perturbation regarded as a solution of the stochastic differential equation (43) corresponds to

$$
\begin{aligned}
\left\langle\Phi_{k}\left(\eta_{1}\right) \Phi_{k^{\prime}}\left(\eta_{2}\right)\right\rangle_{\xi}= & \left\langle\Phi_{k}^{(\mathrm{h})}\left(\eta_{1}\right) \Phi_{k^{\prime}}^{(\mathrm{h})}\left(\eta_{2}\right)\right\rangle_{\xi} \\
& +\left\langle\Phi_{k}^{(\mathrm{h})}\left(\eta_{1}\right) \Phi_{k^{\prime}}^{(\mathrm{i})}\left(\eta_{2}\right)\right\rangle_{\xi} \\
& +\left\langle\Phi_{k}^{(\mathrm{i})}\left(\eta_{1}\right) \Phi_{k^{\prime}}^{(\mathrm{h})}\left(\eta_{2}\right)\right\rangle_{\xi} \\
& +\left\langle\Phi_{k}^{(\mathrm{i})}\left(\eta_{1}\right) \Phi_{k^{\prime}}^{(\mathrm{i})}\left(\eta_{2}\right)\right\rangle_{\xi},
\end{aligned}
$$

where $\langle\ldots\rangle_{\xi}$ denotes the average over all possible realizations of the stochastic source, as previously defined. From now on we will concentrate solely on the last term, which comes entirely from the solutions of the inhomogeneous equation; see Appendix D for a discussion on the role of the initial conditions and the contributions of the homogeneous solution to the correlation function. The correlation function has then the following form:

$$
\begin{aligned}
\left\langle\Phi_{k}^{(\mathrm{i})}\left(\eta_{1}\right) \Phi_{k^{\prime}}^{(\mathrm{i})}\left(\eta_{2}\right)\right\rangle_{\xi} & =\left(\frac{\kappa}{2}\right)^{2} \int_{\eta_{0}}^{\eta_{1}} d \eta_{1}^{\prime} \int_{\eta_{0}}^{\eta_{2}} d \eta_{2}^{\prime} a^{2}\left(\eta_{1}^{\prime}\right) a^{2}\left(\eta_{2}^{\prime}\right) G_{\mathrm{ret}}^{(k)}\left(\eta_{1}, \eta_{1}^{\prime}\right)\left\langle\left(\xi_{0}^{i}\right)_{k}\left(\eta_{1}^{\prime}\right)\left(\xi_{0}^{i}\right)_{k^{\prime}}\left(\eta_{2}^{\prime}\right)\right\rangle_{\xi} G_{\mathrm{ret}}^{\left(k^{\prime}\right)}\left(\eta_{2}, \eta_{2}^{\prime}\right) \\
& =\left(\frac{\kappa}{2}\right)^{2}\left(G_{\mathrm{ret}}^{(k)} \cdot\left(N_{0 i 0 i}\right)_{k k^{\prime}} \cdot\left(G_{\mathrm{ret}}^{\left(k^{\prime}\right)}\right)^{T}\right)\left(\eta_{1}, \eta_{2}\right),
\end{aligned}
$$

where we used the notation $A^{T}\left(\eta, \eta^{\prime}\right)=A\left(\eta^{\prime}, \eta\right)$ and $A$. $B=\int_{\eta_{0}}^{\infty} d \eta A(\eta) B(\eta)$, and the factors $a^{2}\left(\eta_{1}^{\prime}\right)$ and $a^{2}\left(\eta_{2}^{\prime}\right)$ were simplified when lowering the spatial indices with the background metric in the last equality. Note that the final result in Eq. (46) does not involve the stochastic source, which can be regarded just as an intermediate tool to obtain the correlation function of the metric perturbations.

Since we are linearizing in the inflaton perturbations, only the first term on the right-hand side of Eq. (20) should be considered. The expression for the Fourier-transformed version of the noise kernel then becomes

$$
\left(N_{a b c d}\right)_{k k^{\prime}}\left(\eta, \eta^{\prime}\right)=\frac{1}{2}\left\langle\left\{\left(\delta \hat{t}_{a b}\right)_{k}(\eta),\left(\delta \hat{t}_{c d}\right)_{k^{\prime}}\left(\eta^{\prime}\right)\right\}\right\rangle_{\Phi=0},
$$

where $\delta \hat{t}_{a b}=\delta \hat{\mathcal{T}}_{a b}-\left\langle\delta \hat{\mathcal{T}}_{a b}\right\rangle$, as defined earlier, and $\langle\ldots\rangle_{\Phi=0}$ is the expectation value for the product of quantum operators $\delta \hat{t}_{a b}$ with the field $\hat{\varphi}$ evolving on the background metric. We finally obtain the following expression relating the correlation function for the metric perturbations and the fluctuations of the stress tensor operator:

$$
\begin{aligned}
\left\langle\Phi_{k}^{(\mathrm{i})}\left(\eta_{1}\right) \Phi_{k^{\prime}}^{(\mathrm{i})}\left(\eta_{2}\right)\right\rangle_{\xi}= & \left(\frac{\kappa}{2}\right)^{2} \frac{1}{2}\left[G_{\mathrm{ret}}^{(k)} \cdot\left\langle\left\{\left(\delta \hat{t}_{0 i}\right)_{k},\left(\delta \hat{t}_{0 i}\right)_{k^{\prime}}\right\}\right\rangle_{\Phi=0}\right. \\
& \left.\cdot\left(G_{\mathrm{ret}}^{\left(k^{\prime}\right)}\right)^{T}\right]\left(\eta_{1}, \eta_{2}\right) .
\end{aligned}
$$

A detailed example of this kind of computation is given in the next section, where the correlation function of scalartype metric perturbations will be computed for the particular case in which the background solutions for $\phi(\eta)$ and $a(\eta)$ correspond to a period of slow-roll inflation.

We end this subsection by working out the explicit expression for the $0 i$ component of the expectation value $\left\langle\delta \hat{\mathcal{T}}_{a}^{b}\right\rangle_{\Phi}$. From Eq. (31) we get

$$
\left\langle\left(\delta \hat{\mathcal{T}}_{0}^{i}\right)_{k}(\eta)\right\rangle_{\Phi}=i k_{i} a^{-2}(\eta) \phi^{\prime}(\eta)\left\langle\hat{\varphi}_{k}(\eta)\right\rangle_{\Phi},
$$

and everything reduces to compute the expectation value $\left\langle\hat{\varphi}_{k}(\eta)\right\rangle_{\Phi}$. One way of obtaining it is by regarding $\Phi_{k}$ as an external source of the Fourier-transformed version of the linearized Klein-Gordon equation (41) and solving the corresponding inhomogeneous equation perturbatively so that $\left\langle\hat{\varphi}_{k}[g+h]\right\rangle=\left\langle\hat{\varphi}_{k}^{(0)}[g]\right\rangle+\left\langle\hat{\varphi}_{k}^{(1)}[g+h]\right\rangle+O\left(h^{2}\right)$. The expectation value $\left\langle\hat{\varphi}_{k}^{(0)}[g]\right\rangle$ vanishes, and $\left\langle\hat{\varphi}_{k}^{(1)}[g+\right.$ $h]\rangle$ is the solution of the inhomogeneous equation with vanishing initial conditions, which is proportional to the metric perturbation $\Phi_{k}$ and can be written as

$$
\begin{aligned}
\left\langle\hat{\varphi}_{k}^{(1)}(\eta)\right\rangle_{\Phi}= & \int_{\eta_{0}}^{\eta} d \eta^{\prime} \bar{G}_{\mathrm{ret}}^{(k)}\left(\eta, \eta^{\prime}\right)\left\{4 \phi^{\prime}\left(\eta^{\prime}\right) \Phi_{k}^{\prime}\left(\eta^{\prime}\right)\right. \\
& \left.-2 m^{2} a^{2}\left(\eta^{\prime}\right) \phi\left(\eta^{\prime}\right) \Phi_{k}\left(\eta^{\prime}\right)\right\}
\end{aligned}
$$

where $\bar{G}_{\text {ret }}^{(k)}$ is the Fourier-transformed version of the re- 
tarded propagator associated with the Klein-Gordon equation (41) with vanishing initial conditions at $\eta_{0}$. One can show that the retarded propagator $\bar{G}_{\text {ret }}^{(k)}$ for the KleinGordon equation (41) with Fourier-transformed spatial coordinates is given by

$$
\bar{G}_{\text {ret }}^{(k)}\left(\eta, \eta^{\prime}\right)=i a^{2}\left(\eta^{\prime}\right)\left\langle\left[\hat{\varphi}_{k}[g ; \eta), \hat{\varphi}_{-k}\left[g ; \eta^{\prime}\right)\right]\right\rangle \theta\left(\eta-\eta^{\prime}\right) .
$$

Substituting Eq. (51) into Eq. (50) and the result into Eq. (49) one gets the following result for the expectation value $\left\langle\left(\delta \hat{\mathcal{T}}_{0}^{i}\right)_{k}\right\rangle_{\Phi}$ :

$$
\begin{aligned}
\left\langle\left(\delta \hat{\mathcal{T}}_{0}^{i}\right)_{k}(\eta)\right\rangle_{\Phi}= & i k_{i} a^{-2}(\eta) \phi^{\prime}(\eta) \\
& \times \int_{\eta_{0}}^{\eta} d \eta^{\prime} i\left\langle\left[\hat{\varphi}_{k}[g ; \eta), \hat{\varphi}_{-k}\left[g ; \eta^{\prime}\right)\right]\right\rangle \\
& \times a^{2}\left(\eta^{\prime}\right)\left\{4 \phi^{\prime}\left(\eta^{\prime}\right) \Phi_{k}^{\prime}\left(\eta^{\prime}\right)\right. \\
& \left.-2 m^{2} a^{2}\left(\eta^{\prime}\right) \phi\left(\eta^{\prime}\right) \Phi_{k}\left(\eta^{\prime}\right)\right\} .
\end{aligned}
$$

Note that this expression for the expectation value of the stress tensor operator requires no renormalization because we linearized with respect to the scalar field perturbations; see Appendix $\mathrm{C}$ for further comments on this point. Furthermore, in Appendix $\mathrm{C}$ we also show that the expectation value obtained above is in agreement with the general expression for the expectation value of the stress tensor which follows from the approach to the Einstein-Langevin formalism based on functional methods.

\section{Equivalence with the usual quantization methods}

In this subsection we will show that the result for the correlation function of the metric perturbations obtained in the previous subsection using the Einstein-Langevin equation and linearizing in the inflaton perturbations coincides with the result which follows from the usual quantization procedures in linear cosmological perturbation theory; see for instance Refs. [6,75-77].

Let us promote the scalar-type metric perturbations $\Phi$ and the inflaton perturbations $\varphi$ to quantum operators. Equations (33)-(35) then become equations for the operators in the Heisenberg picture. In particular, we will concentrate on the temporal components of the Einstein equation

$$
\begin{gathered}
3 \mathcal{H} \hat{\Phi}^{\prime}+3 \mathcal{H}^{2} \hat{\Phi}-\nabla^{2} \hat{\Phi}=\left.\frac{\kappa}{2} a^{2} \delta \hat{\mathcal{T}}_{0}^{0}\right|_{\hat{\Phi}}, \\
\partial_{i}\left(\hat{\Phi}^{\prime}+\mathcal{H} \hat{\Phi}\right)=\left.\frac{\kappa}{2} a^{2} \delta \hat{\mathcal{T}}_{0}^{i}\right|_{\hat{\Phi}},
\end{gathered}
$$

where the quantum operator for the inflaton perturbations $\hat{\varphi}[g+h]$, on which $\left.\delta \hat{\mathcal{T}}_{a b}\right|_{\hat{\Phi}}$ depends, satisfies the linearized Klein-Gordon equation $\hat{\varphi}^{\prime \prime}+2 \mathcal{H} \hat{\varphi}^{\prime}-\nabla^{2} \hat{\varphi}+m^{2} a^{2} \hat{\varphi}-4 \phi^{\prime} \hat{\Phi}^{\prime}+2 m^{2} a^{2} \phi \hat{\Phi}=0$.

The situation is completely analogous to that of the previous subsection except for the fact that the metric perturbation $\hat{\Phi}(x)$ is now a genuine quantum operator instead of a stochastic $c$-number. Thus, taking the Fourier transform for the spatial coordinates and proceeding in a similar fashion to the previous subsection, the Klein-Gordon equation (55) can be solved with the following result:

$$
\begin{aligned}
\hat{\varphi}_{k}(\eta)= & \hat{\varphi}_{k}^{(0)}(\eta)+\int_{\eta_{0}}^{\eta} d \eta^{\prime} \bar{G}_{\text {ret }}^{(k)}\left(\eta, \eta^{\prime}\right)\left\{4 \phi^{\prime}\left(\eta^{\prime}\right) \hat{\Phi}_{k}^{\prime}\left(\eta^{\prime}\right)\right. \\
& \left.-2 m^{2} a^{2}\left(\eta^{\prime}\right) \phi\left(\eta^{\prime}\right) \hat{\Phi}_{k}\left(\eta^{\prime}\right)\right\},
\end{aligned}
$$

where $\hat{\varphi}_{k}^{(0)}(\eta)$ is a solution of the Klein-Gordon equation on the background spacetime which contains the entire specification of the initial conditions at time $\eta_{0}$ and $\bar{G}_{\text {ret }}^{(k)}$ is the retarded propagator with vanishing initial conditions associated with the Fourier-transformed version of Eq. (55), which coincides with that already obtained for Eq. (41).

Taking into account Eq. (56), one could use any of the constraint equations (53) or (54) to express the quantum operator for the metric perturbation $\hat{\Phi}$ entirely in terms of the operator for the inflaton perturbations $\hat{\varphi}^{(0)}[g]$ [in addition to the scalar functions $\phi(\eta)$ and $a(\eta)$ characterizing the background solution], which satisfies the Klein-Gordon equation on the unperturbed geometry. However, before proceeding further it is convenient to discuss some useful expressions relating $\delta \hat{\mathcal{T}}_{a b}$ and its expectation values on both the background spacetime and the perturbed geometry. The stress tensor operator $\left.\delta \hat{\mathcal{T}}_{a b}\right|_{\hat{\Phi}}$, which is linear in both $\hat{\varphi}[g+h]$ and $\hat{\Phi}$, can actually be written as a linear combination of terms proportional to $\hat{\varphi}^{(0)}[g]$, the inflaton perturbations on the background metric, and terms proportional to $\hat{\Phi}$. The latter correspond to terms coming either from the explicit dependence of the stress tensor on the metric, which gives a local contribution, or from the dependence of $\hat{\varphi}[g+h]$ on the metric perturbations according to Eq. (56). In fact, since Eq. (56) is identical to Eq. (50) when substituting the stochastic function $\Phi_{k}(\eta)$ by the operator $\hat{\Phi}_{k}(\eta)$, it is clear that all the terms proportional to $\hat{\Phi}$ in $\left.\delta \hat{\mathcal{T}}_{a b}\right|_{\hat{\Phi}}$ are identical to the terms proportional to $\Phi$ in the operator $\delta \hat{\mathcal{T}}_{a b}[g+h]$ considered in the previous subsection. Furthermore, since $\left\langle\hat{\varphi}^{(0)}[g]\right\rangle=0$, those terms are identical to $\left\langle\delta \hat{\mathcal{T}}_{a b}\right\rangle_{\hat{\Phi}}$, where $\left\langle\delta \hat{\mathcal{T}}_{a b}\right\rangle_{\hat{\Phi}}$ should be understood as the result of replacing $\Phi$ with $\hat{\Phi}$ in the expectation value $\left\langle\delta \hat{\mathcal{T}}_{a b}\right\rangle_{\Phi}$ of the previous subsection. Hence, we have 


$$
\begin{aligned}
\left.\delta \hat{\mathcal{T}}_{a b}\right|_{\hat{\Phi}}-\left\langle\delta \hat{\mathcal{T}}_{a b}\right\rangle_{\hat{\Phi}} & =\left.\delta \hat{\mathcal{T}}_{a b}\right|_{\hat{\Phi}=0} \\
& =\left.\delta \hat{\mathcal{T}}_{a b}\right|_{\hat{\Phi}=0}-\left\langle\delta \hat{\mathcal{T}}_{a b}\right\rangle_{\hat{\Phi}=0},
\end{aligned}
$$

where we used in the last equality the fact that $\left\langle\delta \hat{\mathcal{T}}_{a b}\right\rangle_{\hat{\Phi}=0}$ actually vanishes. Equation (57) can be written as

$$
\left.\delta \hat{\mathcal{T}}_{a b}\right|_{\hat{\Phi}}=\delta \hat{t}_{a b}+\left\langle\delta \hat{\mathcal{T}}_{a b}\right\rangle_{\hat{\Phi}},
$$

where $\delta \hat{t}_{a b}=\left.\delta \hat{\mathcal{T}}_{a b}\right|_{\hat{\Phi}=0}-\left\langle\delta \hat{\mathcal{T}}_{a b}\right\rangle_{\hat{\Phi}=0}$. It should be remarked that taking the expectation value with respect to some quantum state of the field $\varphi$ should be considered with caution here since, due to the constraint equations (53) and (54), the operators $\hat{\varphi}$ and $\hat{\Phi}$ are not independent. Thus, strictly speaking, $\left\langle\delta \hat{\mathcal{T}}_{a b}\right\rangle_{\hat{\Phi}}$ should be regarded in this context merely as a notation for those terms of $\left.\delta \hat{\mathcal{T}}_{a b}\right|_{\hat{\Phi}}$ which are proportional to $\hat{\Phi}$, in contrast to those proportional to $\hat{\varphi}^{(0)}[g]$.

Substituting Eq. (58) into any of the constraint equations (53) or (54), one could easily obtain the metric perturbation $\hat{\Phi}$ in terms of the operator $\delta \hat{t}_{a b}$ constructed with operators for the inflaton perturbations evolving on the background metric. In order to compare in detail with the result of the previous subsection, where the Einstein-Langevin equation was used, we will explicitly consider the case in which the constraint equation (54) is used. Having substituted $\left.\delta \hat{\mathcal{T}}_{a b}\right|_{\hat{\Phi}}$ by $\delta \hat{t}_{a b}+\left\langle\delta \hat{\mathcal{T}}_{a b}\right\rangle_{\hat{\Phi}}$ into the Fourier-transformed version of Eq. (54), we have

$$
i k_{i}\left(\hat{\Phi}_{k}^{\prime}+\mathcal{H} \hat{\Phi}_{k}\right)-\frac{\kappa}{2} a^{2}\left\langle\left(\delta \hat{\mathcal{T}}_{0}^{i}\right)_{k}\right\rangle_{\hat{\Phi}}=\frac{\kappa}{2} a^{2}\left(\delta \hat{t}_{0}^{i}\right)_{k} .
$$

Taking into account that $\left\langle\delta \hat{\mathcal{T}}_{0}^{i}\right\rangle_{\hat{\Phi}}$ is linear in $\hat{\Phi}$, one can obtain the following expression for $\hat{\Phi}$ in terms of $\delta \hat{t}_{0}^{i}$ from Eq. (59):

$$
\hat{\Phi}_{k}(\eta)=\hat{\Phi}_{k}^{(\mathrm{h})}(\eta)+\hat{\Phi}_{k}^{(\mathrm{i})}(\eta)
$$

where $\hat{\Phi}_{k}^{(\mathrm{h})}$ is a solution of the homogeneous version of Eq. (59) and

$$
\begin{aligned}
\hat{\Phi}_{k}^{(\mathrm{i})}(\eta) & =\frac{\kappa}{2} \int_{\eta_{0}}^{\eta} d \eta^{\prime} a^{2}\left(\eta^{\prime}\right) G_{\mathrm{ret}}^{(k)}\left(\eta, \eta^{\prime}\right)\left(\delta \hat{t}_{0}^{i}\right)_{k}\left(\eta^{\prime}\right) \\
& =\frac{\kappa}{2}\left(G_{\mathrm{ret}}^{(k)} \cdot\left(\delta \hat{t}_{0 i}\right)_{k}\right)(\eta),
\end{aligned}
$$

where $G_{\text {ret }}^{(k)}$ is the retarded Green function and the factor $a^{2}\left(\eta^{\prime}\right)$ canceled out when lowering the spatial index $i$ with the background metric. In fact, since the homogeneous part of Eq. (59) has exactly the same form as that of Eq. (43), the retarded Green function $G_{\text {ret }}^{(k)}$ coincides with that of the previous subsection. An analogous remark holds for the solution of the homogeneous equation $\hat{\Phi}_{k}^{(\mathrm{h})}$.
Using Eq. (61) and concentrating on the inhomogeneous contribution (a discussion of the homogeneous solution and its relationship to the initial conditions is given in Appendix D), the symmetrized two-point quantum correlation function for the metric perturbation operator $\hat{\Phi}$ can be written as

$$
\begin{aligned}
\frac{1}{2}\left\langle\left\{\hat{\Phi}_{k}^{(\mathrm{i})}\left(\eta_{1}\right), \hat{\Phi}_{k^{\prime}}^{(\mathrm{i})}\left(\eta_{2}\right)\right\}\right\rangle= & \left(\frac{\kappa}{2}\right)^{2} \frac{1}{2}\left[G_{\mathrm{ret}}^{(k)}\right. \\
& \cdot\left\langle\left\{\left(\delta \hat{t}_{0 i}\right)_{k},\left(\delta \hat{t}_{0 i}\right)_{k^{\prime}}\right\}\right\rangle_{\Phi=0} \\
& \left.\cdot\left(G_{\mathrm{ret}}^{\left(k^{\prime}\right)}\right)^{T}\right]\left(\eta_{1}, \eta_{2}\right) .
\end{aligned}
$$

Thus, we can see that the result for the symmetrized quantum correlation function of the metric perturbations coincides with the stochastic correlation function (48) obtained in the previous subsection using the EinsteinLangevin equation.

We end this section by making a few remarks concerning the issue of the normalization of cosmological perturbations. In principle, one could differentiate Eq. (54) with respect to the conformal time (the spatial derivatives can be easily eliminated by working in Fourier space) and combine it with Eq. (53) to obtain a linear second-order differential equation for the metric perturbation operator $\hat{\Phi}$. However, when trying to quantize a theory beginning with the equations of motion instead of an action, one faces a normalization ambiguity which stems from the fact that, although any pair of actions that differ in a constant factor yield the same equations of motion (either classical or for quantum operators in the Heisenberg picture), their corresponding quantum theories are not completely equivalent. In particular, the quantum correlation functions for a given state (e.g. the fundamental state) do not coincide. For a linear theory they actually differ by some power of a constant factor which is precisely the square root of the proportionality constant between the two actions. This is the reason why in Ref. [76], when quantizing the theory for linear perturbations, the final action was obtained from the original linearized action for a scalar field evolving on a metric perturbed around a given background geometry together with the linearized EinsteinHilbert action for the perturbations of that metric. That was done by using the constraint equations to reduce the whole action to that for the only true dynamical degrees of freedom. It was precisely in order to avoid the normalization ambiguity explained above that such a procedure, which turns out to be rather cumbersome, was used instead of working directly with the equations of motion and finding at the end an action which corresponds to the equation of motion for the true dynamical degree of freedom.

On the other hand, the method employed in this section is not affected by such a normalization ambiguity because, as can be seen from Eqs. (30) and (54), the constraint 
equation relates the operator for the metric perturbations to the operator for the inflaton perturbations on the background metric. The normalization of the latter operator is already determined by the usual procedure of quantization starting from the action of a scalar field on a fixed spacetime geometry. Hence, the key point was to separate the inflaton perturbation operator $\hat{\varphi}$ satisfying the KleinGordon equation (55) into a contribution $\hat{\varphi}^{(0)}[g]$ which can be regarded as the inflaton perturbation evolving on the fixed background spacetime plus a contribution proportional to the metric perturbation operator $\hat{\Phi}$, and use then the constraint equation to express $\hat{\Phi}$ entirely in terms of $\hat{\varphi}^{(0)}[g]$. In contrast with the approach of Ref. [76], this procedure does not give an explicit expression for the reduced action or even the equation of motion for an isolated true dynamical degree of freedom but it is rather useful (and sufficient) in order to compare with the results obtained in the previous subsection by means of the Einstein-Langevin equation.

\section{PARTICULAR EXAMPLE: COMPUTATION OF THE POWER SPECTRUM FOR LARGE SCALES IN A SIMPLE INFLATIONARY MODEL}

In this section we will apply the method developed in Sec. III B to study the particular example of metric fluctuations induced by the quantum fluctuations of the inflaton field in the context of a simple model of chaotic inflation corresponding to a free minimally-coupled massive scalar field. In order to carry out explicit computations, we will assume that the Robertson-Walker background geometry is close to the de Sitter geometry. For models with exponential inflation, as the one being considered here, this approximation is reasonable during the inflationary period, in which the so-called slow-roll parameters controlling the deviations from de Sitter geometry are small, but not for later times. One can, nevertheless, obtain useful results from a cosmological point of view because those scales which are of cosmological interest at present correspond to scales which left the horizon during the inflationary period. This can be understood as follows. On the one hand, the evolution of gravitational perturbations outside the horizon is fairly simple, as can be understood from causality arguments $[6,12,76]$, and rather independent of the particular dynamics of the matter sources. On the other hand, the evolution when the scale reenters the horizon later on during the radiation and matter dominated eras has been widely studied using the Newtonian approximation [78,79].

We stress that the results obtained in this section, which are based on the use of the Einstein-Langevin equation, are not new. They are basically in agreement with most of the literature based on the simultaneous quantization of gravitational perturbations and inflaton perturbations when both are treated linearly. ${ }^{3}$ Of course, this fact ultimately follows from the equivalence between both approaches established in Sec. III C (as well as in Appendix E). Thus, the purpose of this section is to illustrate with a simple but relevant example how the Einstein-Langevin equation can be useful to obtain explicit results concerning cosmological perturbations.

Let us start by recalling the expression for the $0 i$ component of the Einstein-Langevin equation which was obtained in Sec. III B working in Fourier space for the spatial components:

$$
i k_{i}\left(\Phi_{k}^{\prime}+\mathcal{H} \Phi_{k}\right)=\frac{\kappa}{2} a^{2}\left(\left\langle\left(\delta \hat{\mathcal{T}}_{0}^{i}\right)_{k}\right\rangle_{\Phi}+\left(\xi_{0}^{i}\right)_{k}\right) .
$$

The expectation value of the linearized stress tensor operator is given by Eq. (52) and is nonlocal in the conformal time. In general, this fact makes it difficult to find an analytic expression for the solution of the EinsteinLangevin equation.

One possible approach is to realize that there is a certain linear combination of the different components of the Einstein-Langevin equation for which all the contributions from the nonlocal terms cancel out, as well as those from the stochastic source (a detailed proof and discussion is provided in Appendix E). In that case the equation that one needs to solve, Eq. (E5), is a linear second-order ordinary differential equation. In fact, this equation has the same form as one often considered in standard treatments of linearized cosmological perturbations (see Eq. (6.48) in Ref. [76]), and one can take advantage of the existing methods and approximation schemes for solving it. Nevertheless, for illustrative purposes we will not follow this approach in this section. We will directly consider Eq. (63), neglect its nonlocal part, and concentrate on the fluctuating part (neglecting the nonlocal term is not necessary but it simplifies the problem considerably for a quick calculation). ${ }^{4}$ Eq. (63) then becomes

\footnotetext{
${ }^{3}$ More specifically, we will obtain a Harrison-Zeldovich spectrum for the scalar metric perturbations with an amplitude which has the right dependence on the parameters of the problem (the Planck mass and the mass of the quadratic inflaton potential). However, our simple calculation does not give the right result for the spectral index: it gives a spectral index whose value is exactly 1 (rather than slightly smaller than 1). In fact, one can explicitly check that the three main approximations that will be employed in this section (namely, neglecting the nonlocal terms, considering a de Sitter background, and computing the quantum correlation function for the inflaton field using the massless approximation) all contribute to a comparable deviation from the exact result for the spectral index, whereas the correct result is obtained when none of the approximations are made. (All this can be checked by proceeding analogously to the calculation in Sec. 8.2.2 of Ref. [77].)

${ }^{4}$ One can see from our derivations in Sec. III that neglecting the nonlocal term is equivalent to neglecting the terms proportional to the metric in Eq. (41).
} 


$$
2 i k_{i}\left(\mathcal{H} \Phi_{k}+\Phi_{k}^{\prime}\right) \simeq \frac{8 \pi}{m_{p}^{2}}\left(\xi_{0 i}\right)_{k}
$$

from which we can obtain the metric perturbation $\Phi_{k}$ in terms of the stochastic source $\left(\xi_{0 i}\right)_{k}$. We need the retarded propagator for the gravitational potential $\Phi_{k}$, i.e., the required Green function to solve the inhomogeneous firstorder differential equation (64) with the appropriate boundary conditions:

$$
G_{k}^{\mathrm{ret}}\left(\eta, \eta^{\prime}\right)=-\frac{i}{2 k_{i}}\left(\theta\left(\eta-\eta^{\prime}\right) \frac{a\left(\eta^{\prime}\right)}{a(\eta)}+f\left(\eta, \eta^{\prime}\right)\right)
$$

where $f\left(\eta, \eta^{\prime}\right)$ is a homogeneous solution related to the chosen initial conditions. In particular, if we take $f\left(\eta, \eta^{\prime}\right)=-\theta\left(\eta_{0}-\eta^{\prime}\right) a\left(\eta^{\prime}\right) / a(\eta)$, we have $G_{k}^{\text {ret }}\left(\eta, \eta^{\prime}\right)=0$ for $\eta \leq \eta_{0}$, which gives the stochastic evolution of the metric perturbations for $\eta>\eta_{0}$ due to the effect of the stochastic source after $\eta_{0}$. The correlation function for the metric perturbations is then given by the following expression:

$$
\begin{aligned}
\left\langle\Phi_{k}(\eta) \Phi_{k^{\prime}}\left(\eta^{\prime}\right)\right\rangle_{\xi} \simeq & \left(\frac{8 \pi}{m_{p}^{2}}\right)^{2} \int_{\eta_{0}}^{\eta} d \eta_{1} \int_{\eta_{0}}^{\eta^{\prime}} d \eta_{2} G_{k}^{\mathrm{ret}}\left(\eta, \eta_{1}\right) \\
& \times G_{k^{\prime}}^{\mathrm{ret}}\left(\eta^{\prime}, \eta_{2}\right)\left\langle\xi_{k 0 i}\left(\eta_{1}\right) \xi_{k^{\prime} 0 i}\left(\eta_{2}\right)\right\rangle_{\xi}
\end{aligned}
$$

The correlation function for the stochastic source is, in turn, connected with the stress tensor fluctuations:

$$
\begin{aligned}
\left\langle\xi_{k 0 i}\left(\eta_{1}\right) \xi_{k^{\prime} 0 i}\left(\eta_{2}\right)\right\rangle_{\xi}= & \frac{1}{2}\left\langle\left\{\hat{t}_{0 i}^{k}\left(\eta_{1}\right), \hat{t}_{0 i}^{k^{\prime}}\left(\eta_{2}\right)\right\}\right\rangle_{\phi \varphi} \\
= & (2 \pi)^{3} \delta\left(\vec{k}+\vec{k}^{\prime}\right) \\
& \times \frac{1}{2} k_{i} k_{i} \phi^{\prime}\left(\eta_{1}\right) \phi^{\prime}\left(\eta_{2}\right) G_{k}^{(1)}\left(\eta_{1}, \eta_{2}\right),
\end{aligned}
$$

where the delta function follows from spatial translational invariance and $G_{k}^{(1)}\left(\eta_{1}, \eta_{2}\right)=\left\langle\left\{\hat{\varphi}_{k}\left(\eta_{1}\right), \hat{\varphi}_{-k}\left(\eta_{2}\right)\right\}\right\rangle$ is the $k$-mode Hadamard function for a free minimally-coupled scalar field which is in a state close to the Bunch-Davies vacuum on an almost de Sitter background. The so-called slow-roll parameters account for the fact that the background geometry is not exactly that of de Sitter spacetime, for which $a(\eta)=-1 / H \eta$ with $-\infty<\eta<0$.

It is also useful to compute the Hadamard function for a massless field and consider a perturbative expansion in terms of the dimensionless parameter $m / m_{p}$, for which observations seem to imply, as will be seen below, a value of the order of $10^{-6}$. Thus, we will consider $\bar{G}_{k}^{(1)}\left(\eta_{1}, \eta_{2}\right)=$ $a\left(\eta_{1}\right) a\left(\eta_{2}\right) G_{k}^{(1)}\left(\eta_{1}, \eta_{2}\right)=\left\langle 0\left|\left\{\hat{y}_{k}\left(\eta_{1}\right), \hat{y}_{-k}\left(\eta_{2}\right)\right\}\right| 0\right\rangle \quad$ such that $\hat{a}_{k}|0\rangle=0 \quad$ with $\hat{y}_{k}(\eta)=a(\eta) \hat{\varphi}_{k}(\eta)=\hat{a}_{k} u_{k}(\eta)+$ $\hat{a}_{-k}^{\dagger} u_{-k}^{*}(\eta)$ and $u_{k}(\eta)=(2 k)^{-1 / 2} e^{-i k \eta}(1-i / k \eta)$ corresponding to the positive frequency $k$-mode for a massless minimally-coupled scalar field in the Bunch-Davies vacuum state on a de Sitter background [42].

The result to lowest order in the mass $m$ of the inflaton field and the slow-roll parameters is:

$$
\begin{aligned}
\left\langle\Phi_{k}(\eta) \Phi_{k^{\prime}}\left(\eta^{\prime}\right)\right\rangle_{\xi} \simeq & \frac{64 \pi^{5}}{m_{p}^{4}} \delta\left(\vec{k}+\vec{k}^{\prime}\right) \int_{\eta_{0}}^{\eta} d \eta_{1} \int_{\eta_{0}}^{\eta^{\prime}} d \eta_{2} \frac{a\left(\eta_{1}\right)}{a(\eta)} \frac{a\left(\eta_{2}\right)}{a\left(\eta^{\prime}\right)} \dot{\phi}\left(\eta_{1}\right) \dot{\phi}\left(\eta_{2}\right) \bar{G}_{k}^{(1)}\left(\eta_{1}, \eta_{2}\right) \\
= & 64 \pi^{5}\left(\frac{m}{m_{p}}\right)^{2} k^{-3} \delta\left(\vec{k}+\vec{k}^{\prime}\right) \int_{k \eta_{0}}^{k \eta} d\left(k \eta_{1}\right) \int_{k \eta_{0}}^{k \eta^{\prime}} d\left(k \eta_{2}\right) \frac{k \eta}{k \eta_{1}} \frac{k \eta^{\prime}}{k \eta_{2}} \\
& \times\left[\left(1+\frac{1}{k \eta_{1} k \eta_{2}}\right) \cos k\left(\eta_{1}-\eta_{2}\right)-\left(\frac{1}{k \eta_{1}}-\frac{1}{k \eta_{2}}\right) \sin k\left(\eta_{1}-\eta_{2}\right)\right] \\
= & 64 \pi^{5}\left(\frac{m}{m_{p}}\right)^{2} k^{-3} \delta\left(\vec{k}+\vec{k}^{\prime}\right)\left[\cos k\left(\eta-\eta^{\prime}\right)-\frac{1}{k \eta_{0}}\left(k \eta \cos k\left(\eta-\eta_{0}\right)+k \eta^{\prime} \cos k\left(\eta^{\prime}-\eta_{0}\right)\right)+\frac{k \eta k \eta^{\prime}}{\left(k \eta_{0}\right)^{2}}\right],
\end{aligned}
$$

where we used the lowest order approximation for $\dot{\phi}(t)$ during slow-roll: $\dot{\phi}(t) \simeq-m_{p}^{2}\left(m / m_{p}\right)$; overdots denote here derivatives with respect to the physical time $t$. We considered the effect of the stochastic source after the conformal time $\eta_{0}$. Notice that the result (68) is rather independent of the value of $\eta_{0}$ provided that it is negative enough, i.e., it corresponds to an early enough initial time. This weak dependence on the initial conditions is fairly common in this context and can be qualitatively understood as follows: after a sufficient amount of time, the accelerated expansion for the quasi-de Sitter spacetime during inflation effectively erases any information about the initial conditions, which is redshifted away. The actual result will, therefore, be very close to that for $\eta_{0}=-\infty$ :

$$
\begin{aligned}
\left\langle\Phi_{k}(\eta) \Phi_{k^{\prime}}\left(\eta^{\prime}\right)\right\rangle_{\xi} \simeq & 8 \pi^{2}\left(\frac{m}{m_{p}}\right)^{2} k^{-3}(2 \pi)^{3} \delta\left(\vec{k}+\vec{k}^{\prime}\right) \\
& \times \cos k\left(\eta-\eta^{\prime}\right) .
\end{aligned}
$$

One remark concerning the massless approximation for the computation of the Hadamard function of the inflaton perturbations is needed. It is clear from the equation for the 
scalar field modes that when one considers scales much smaller than the Compton wavelength of the inflaton field, i.e., $k / a(\eta) \gg m$, the effect of the mass term can be neglected. On the other hand, for scales larger than the Compton wavelength one could object that the mass term should no longer be negligible. However, it can be argued that the mass term can also be neglected for large scales provided that the Compton wavelength is much larger than the horizon (the Hubble radius $H^{-1}$ ), i.e., $H \gg m$. The argument goes as follows. For a massless minimallycoupled scalar field in de Sitter spacetime the modes become effectively frozen after leaving the horizon: $k / a(\eta)<H$. On the other hand, for a massive scalar field, the modes decay approximately like $\exp \left(-m^{2} \Delta t / 3 H\right)$ outside the horizon but this decay will not be important if $\mathrm{m}^{2} / \mathrm{H}^{2}$ is small enough. In particular, if $3 H^{2} / \mathrm{m}^{2} \geq 60$, the decay factor $\exp \left[-\left(\mathrm{m}^{2} / 3 H^{2}\right) H \Delta t\right]$ will not be too different from one for those modes that left the horizon during the last $60 e$-folds of inflation $(H \Delta t=60$ with $\Delta t$ being the time between horizon exit and the end of inflation), which includes all the relevant cosmological scales since the scale that left the horizon $60 e$-folds before the end of inflation corresponds to the size of the visible Universe at present; any feature with a scale larger than the visible Universe appears to us as observationally indistinguishable from a homogeneous one. Hence, due to the special behavior of the modes outside the horizon, even when considering scales which became much larger than the Compton wavelength before the end of inflation, $k \exp (-H \Delta t)<m$, it is reasonable to approximate a massive scalar field with a massless one as long as $H \gg m$, which happens to be the case in most slow-roll inflationary models and, in particular, for the simple example considered in this section [5].

Let us consider the cosmological implications which can be extracted from Eq. (69), especially those related to large-scale gravitational fluctuations. These fluctuations are believed to play a crucial role in the generation of the large-scale structure and matter distribution observed in our present Universe [79]. They are also closely connected with the anisotropies in the cosmic microwave background $(\mathrm{CMB})$ radiation, which decoupled from matter about $4 \times$ $10^{5}$ years after the big bang and provides us with very valuable information about the early Universe $[6,77,78]$.

From the analysis of our final result in Eq. (69) two main well-known facts can be concluded. First, an almost Harrison-Zeldovich scale-invariant spectrum is obtained for large scales. Indeed, for scales clearly outside the horizon at the times $\eta$ and $\eta^{\prime}$, i.e., $k \eta, k \eta^{\prime} \ll 1$, the right-hand side of Eq. (69) becomes proportional to $k^{-3} \delta\left(\vec{k}+\vec{k}^{\prime}\right)$ with negligible extra dependence on $k, \eta$, and $\eta^{\prime}$. Second, since we get $\left\langle\Phi_{k}(\eta) \Phi_{k^{\prime}}\left(\eta^{\prime}\right)\right\rangle_{\xi} \propto\left(m / m_{p}\right)^{2}$ in agreement with the usual results $[23,76,77]$, the small value of the $\mathrm{CMB}$ anisotropies first detected by COBE imposes a severe bound on the gravitational fluctuations, characterized by $\left\langle\Phi_{k}(\eta) \Phi_{k^{\prime}}\left(\eta^{\prime}\right)\right\rangle_{\xi}$, which implies the fol- lowing restriction (fine-tuning) for the inflaton mass: $m / m_{p} \sim 10^{-6}$.

Some comments on the mechanisms considered in earlier related work [80-83] which allowed a significant relaxation on the fine-tuning of that kind of parameter are in order here. In those studies either a self-interacting scalar field or a scalar field interacting nonlinearly with other fields were considered. The modes of the inflaton field corresponding to scales of cosmological interest were regarded as an open quantum system with the environment constituted either by the short-wavelength modes in the case of self-interaction or else by other fields interacting with the inflaton field. Therefore, one can introduce a stochastic description based on a Langevin equation, as explained in Ref. [33], to study the dynamics of the inflaton field modes. In fact, Langevin-type equations or related stochastic tools were employed in the references cited above. Furthermore, it was shown in Ref. [33] that the validity of the results, such as the correlation functions, obtained by those methods is independent of the existence of enough decoherence to guarantee the presence of a semiclassical regime for the system dynamics. However, it was also shown that the two-point quantum correlation function for the system had two separate contributions (see Eq. (4.9) in Ref. [33]): one related to the dispersion of the system's initial state and another one which was proportional to the noise kernel and accounted for the fluctuations of the system induced by the interaction with the environment. For natural states in de Sitter spacetime such as the Bunch-Davies vacuum (in fact, any reasonable state in de Sitter space tends asymptotically to it [84]) the dispersion is proportional to $H^{2}$. This contribution is actually several orders of magnitude larger than that coming from the term proportional to the noise kernel for the situations considered in Refs. [80-83]. Thus, the two-point quantum correlation function for the inflaton perturbations is dominated by the contribution connected to the dispersion of the initial state. This point has been confirmed by a detailed analysis in Ref. [24]. Moreover, this contribution essentially coincides for the cases of an interacting and a free scalar field. The latter is the case being considered throughout this paper and exhibits no noise term for the inflaton dynamics because there is no environment for the inflaton perturbations (this should not be confused with the noise kernel for the fluctuations of the metric perturbations induced by the quantum fluctuations of the inflaton). One could try to choose the initial state, as argued in Ref. [83], so that the contribution from the dispersion of the initial conditions were smaller than the fluctuations induced on the modes of the inflaton field, but that would require a great amount of fine-tuning for the initial quantum state of each mode, which would become highly unstable due to the large dispersion in momentum implied by Heisenberg's uncertainty principle [23] and quickly tend to the de Sitter invariant Bunch-Davies vacuum; or even have no inflation 
at all due to the backreaction on the evolution of the background geometry generated by such a highly excited state.

\section{DISCUSSION}

In this paper we have studied linearized metric perturbations around a Robertson-Walker background interacting with a quantum scalar field and we have shown that, when linearizing the perturbations of the scalar field around its background configuration, the EinsteinLangevin equation yields a result for the correlation function of the metric perturbations equivalent to that obtained in the usual approach based on the linearization and quantization of both the metric perturbations and the perturbations of the scalar field around its expectation value. Although for the sake of concreteness we have mostly concentrated on the case of a spatially-flat RobertsonWalker metric and a minimally-coupled scalar field with a quadratic potential, the main result can be generalized rather straightforwardly to Robertson-Walker metrics with nonflat homogeneous spatial sections, as well as to general potentials for the scalar field and arbitrary coupling to the spacetime curvature. Considering Robertson-Walker metrics with homogeneous spatial sections of positive or negative curvature would imply, respectively, the use of threedimensional spherical or hyperbolic harmonics rather than simple Fourier transforms for the spatial coordinates, but that would not substantially change the procedures and the main conclusions since the basic properties of Fourier transforms employed in the text have analogous counterparts for these harmonics [85-87]. On the other hand, the use of a general potential should not imply major differences since after all we would linearize with respect to the scalar field perturbations around the background configuration.

In addition, we also provided in Sec. IV a particular example illustrating how the Einstein-Langevin equation can be used in practice to compute the correlation function at large scales for scalar metric perturbations in cosmological inflationary models. In doing so we made use of slightly oversimplified approximations, namely, use of a de Sitter background geometry, calculation of the Hadamard function for a massless scalar field and neglecting a nonlocal term, because the result has already been computed in a number of references, see for instance Refs. [5,6,75-77], and our primary concern was simply to show how the Einstein-Langevin equation can be used to obtain an explicit result for cosmological perturbations.

Throughout the article we have concentrated on scalartype metric perturbations. The reason for this is that, when linearizing with respect to both the metric perturbations and the scalar field perturbations, the vectorial and tensorial metric perturbations decouple from the matter scalar field. In this case the metric perturbations do not constitute a true open system since the dynamics of the scalar and vectorial perturbations is completely constrained by the temporal components of the Einstein equation; in fact, the vectorial ones actually turn out to vanish and the scalar ones cannot be regarded as a degree of freedom independent of the scalar field perturbations. Moreover, the only true dynamical degrees of freedom, the two tensorial ones, do not couple to the matter field. On the other hand, an even more interesting situation corresponds to the case in which the scalar field is treated exactly, at least for quadratic potentials. Then the scalar field also couples to the metric perturbations of vectorial and tensorial type and the metric perturbations become a true open system with the scalar field corresponding to the environment.

The main features that would characterize an exact treatment of the scalar field perturbations interacting with the metric perturbations around a Robertson-Walker background as compared to the case addressed in this paper are the following. First, the three types of metric perturbations couple to the perturbations of the scalar field, as already mentioned above. Second, although the Fourier modes (with respect to the spatial coordinates) for the metric perturbations will still decouple in the Einstein-Langevin equation, any given mode of the noise and dissipation kernels will get contributions from an infinite number of Fourier modes of the scalar field perturbations (see Ref. [88] for an explicit calculation of the noise kernel for a massless and minimally-coupled scalar field in de Sitter). This fact will imply, in addition, the need to properly renormalize the ultraviolet divergences arising in the dissipation kernel, which actually correspond to the divergences associated with the expectation value of the stress tensor operator of the quantum matter field evolving on the perturbed geometry.

The importance of considering corrections due to oneloop contributions from scalar field perturbations, beyond the tree level of the linear cosmological perturbation theory, has recently been emphasized [29,30]. In the context of stochastic gravity this means treating the scalar field perturbations exactly in the Einstein-Langevin equation. Furthermore, in Ref. [33] it was explained how a stochastic description based on a Langevin-type equation could be introduced to gain information on fully quantum properties of simple linear open systems. In a forthcoming paper [36] it will be shown that, by carefully dealing with the gauge freedom and the consequent dynamical constraints, the previous result can be extended to the case of $N$ free quantum matter fields weakly interacting with the metric perturbations around a given background (here weakly interacting means that the gravitational coupling constant times the number of fields remains constant in the limit of large $N$ ). In particular, the correlation functions for the metric perturbations obtained using the Einstein-Langevin equation are equivalent to the leading order contribution in the large $N$ limit to the correlation functions that would follow from a purely quantum field theory calculation. This 
will generalize the results already obtained on a Minkowski background [37,38]. These results have important implications on the use of the Einstein-Langevin equation to address situations in which the background configuration for the scalar field vanishes, so that linearization around such a configuration is no longer possible. This includes not only the case of a Minkowski background spacetime, but also the remarkably interesting case of inflationary models driven by the vacuum polarization of a large number of conformal fields with vanishing expectation value $[32,89,90]$, where the usual approaches based on the linearization of both the metric perturbations and the scalar field perturbations and their subsequent quantization can no longer be applied.

\section{ACKNOWLEDGMENTS}

We are grateful to Daniel Arteaga, Esteban Calzetta, Antonio Campos, Jaume Garriga, Bei-Lok Hu, Kei-ichi Maeda, Rosario Martín and Yuko Urakawa for many interesting discussions. This work has been partially supported by the Research Projects MEC FPA-2004-04582, FPA-2007-66665 and DURSI 2005SGR00082. During the last stages of this project A. R. has also been supported by LDRD funds from Los Alamos National Laboratory.

\section{APPENDIX A: DEFINITION AND BASIC PROPERTIES OF GAUSSIAN STATES}

In this appendix we summarize the definition and basic properties of Gaussian pure states. In contrast to the rest of the paper, $\Psi$ will be used throughout this appendix to denote either the state of a field or its wave functional in the Schrödinger picture rather than a gauge-invariant variable for scalar-type metric perturbations.

A pure state is called Gaussian if its wave functional in the Schrödinger picture is a Gaussian functional:

$$
\begin{gathered}
\Psi[\phi(\vec{x})] \propto \exp \left[-\int d^{3} x d^{3} x^{\prime} \phi(\vec{x}) A\left(\vec{x}, \vec{x}^{\prime}\right) \phi\left(\vec{x}^{\prime}\right)\right. \\
\left.+\int d^{3} x B(\vec{x}) \phi(\vec{x})\right],
\end{gathered}
$$

with a suitable normalization constant. The fundamental property of Gaussian states is the fact that the cumulants of order higher than two associated with the quantum expectation values of products of the field operator $\hat{\phi}$ vanish, i.e.,

$$
\left.\frac{1}{i^{n}} \frac{\delta}{\delta j\left(\vec{x}_{1}\right)} \ldots \frac{\delta}{\delta j\left(\vec{x}_{n}\right)} \ln \left\langle\exp ^{i \int d^{3} x j(\vec{x}) \hat{\phi}(\vec{x})}\right\rangle_{\Psi}\right|_{j=0}=0,
$$

for $n \geq 3$ and where we introduced the notation $\langle\hat{O}\rangle_{\Psi} \equiv$ $\langle\Psi|\hat{O}| \Psi\rangle$. This implies that the connected part of any quantum correlation function $\left\langle\Psi\left|\hat{\phi}\left(\vec{x}_{n}\right) \ldots \hat{\phi}\left(\vec{x}_{1}\right)\right| \Psi\right\rangle$ with $n \geq 3$ vanishes or, equivalently, that any quantum correlation function $\left\langle\Psi\left|\hat{\phi}\left(\vec{x}_{n}\right) \ldots \hat{\phi}\left(\vec{x}_{1}\right)\right| \Psi\right\rangle$ can be written as a linear combination of products involving the expecta- tion values $\left\langle\Psi\left|\hat{\phi}\left(\vec{x}_{i}\right)\right| \Psi\right\rangle$ and two-point functions $\left\langle\Psi\left|\hat{\phi}\left(\vec{x}_{j}\right) \hat{\phi}\left(\vec{x}_{k}\right)\right| \Psi\right\rangle$. Furthermore, if the Hamiltonian of the field under consideration is quadratic, this property can be generalized for different times to quantum correlation functions in the Heisenberg picture of the form ${ }_{H}\left\langle\Psi\left|\hat{\phi}\left(t_{n}, \vec{x}_{n}\right) \ldots \hat{\phi}\left(t_{1}, \vec{x}_{1}\right)\right| \Psi\right\rangle_{H}$, which follows from Wick's theorem [91].

Finally, given a field operator $\hat{\phi}$ and a Gaussian state $|\Psi\rangle$ with nonvanishing expectation value $\langle\hat{\phi}\rangle_{\Psi}$, it is always possible to introduce a new field $\hat{\varphi}=\hat{\phi}-\langle\hat{\phi}\rangle_{\Psi}$ so that the wave functional $\tilde{\Psi}[\varphi]$ for the state $|\Psi\rangle$ in the basis associated with the field $\hat{\varphi}$ becomes a Gaussian functional with vanishing expectation value and independent of the expectation value $\langle\hat{\phi}\rangle_{\Psi}$. This can be immediately seen by rewriting the expression for the wave functional in Eq. (A1) as

$$
\begin{aligned}
\Psi[\phi(\vec{x})] \propto & \exp \left[-\int d^{3} x d^{3} x^{\prime}(\phi(\vec{x})\right. \\
& \left.\left.-\langle\hat{\phi}(\vec{x})\rangle_{\Psi}\right) A\left(\vec{x}, \vec{x}^{\prime}\right)\left(\phi\left(\vec{x}^{\prime}\right)-\langle\hat{\phi}(\vec{x})\rangle_{\Psi}\right)\right],
\end{aligned}
$$

and then changing to the basis associated with the field $\hat{\varphi}$ :

$$
\tilde{\Psi}[\varphi(\vec{x})] \propto \exp \left[-\int d^{3} x d^{3} x^{\prime} \varphi(\vec{x}) A\left(\vec{x}, \vec{x}^{\prime}\right) \varphi\left(\vec{x}^{\prime}\right)\right] .
$$

It is precisely in this sense that the state for the inflaton field perturbations $\hat{\varphi}$ introduced in Sec. II B follows immediately from the state of the inflaton field $\hat{\phi}$.

It should be emphasized that any of the vacuum states commonly considered for free fields in curved spacetimes are Gaussian states. Furthermore, as stated in Sec. II B, in this paper we concentrate on states which are invariant under the symmetries of the Robertson-Walker metric. In particular, for the case of Robertson-Walker metrics with flat spatial sections this implies $\langle\hat{\phi}\rangle_{\Psi}(t, \vec{x})=\langle\hat{\phi}\rangle_{\Psi}(t)$ and $A\left(\vec{x}, \vec{x}^{\prime}\right)=A\left(\left|\vec{x}-\vec{x}^{\prime}\right|\right)$.

\section{APPENDIX B: CONVENTIONS AND NOTATION FOR THE LINEARIZED STRESS TENSOR}

Here we explain the notation concerning the linearized stress tensor employed in this article and clarify some related subtle points.

Let us begin with the objects which appear in the Einstein-Langevin equation (7). The tensors $G_{a b}^{(1)}$ and $T_{a b}^{(1)}$ correspond to the terms proportional to the metric perturbations in the perturbed version of the background (unperturbed) objects $G_{a b}^{(0)}$ and $T_{a b}^{(0)}$. The indices of $G_{a b}^{(1)}$ can be raised using the background metric [from now on everything that will be said for $G_{a b}^{(1)}$ applies exactly in the same way to $\left.T_{a b}^{(1)}\right]$. On the other hand, one could perturb the background object $G^{(0) a b}$ with the indices already raised, and reach a different result for $G^{(1) a b}$ : it would differ by the terms $G^{(0) c d} h_{c}^{a}+G^{(0) a c} h_{c}^{b}$. Our notation (and those com- 
monly employed) is ambiguous in the sense that it does not distinguish between both possibilities. In order to remove such an ambiguity it is necessary (and sufficient) to specify a priori which objects are going to be perturbed. In particular, in Sec. II it is $G_{a b}^{(0)}$ and $T_{a b}^{(0)}$ that are perturbed (of course, one can then freely use the background metric to raise and lower indices), whereas it is $G_{a}^{(0) b}$ and $T_{a}^{(0) b}$ that will be perturbed in Secs. III and IV, and finally $G^{(0) a b}$ and $T^{(0) a b}$ in Appendix D. Fortunately, this will not change the form of the Einstein-Langevin equation since the terms corresponding to the difference between both choices for the Einstein tensor and the stress tensor cancel out because their background counterparts satisfy the Einstein equation $G_{a b}^{(0)}=\kappa T_{a b}^{(0)}$. Hence, if one deals with equations involving tensorial objects rather than with isolated objects, everything is independent of the particular choice provided that the same choice is made for all the objects in the equation.

The previous ambiguity does not affect the stochastic source of the Einstein-Langevin equation, which is completely defined on the background spacetime (the noise kernel is evaluated on the background geometry). Furthermore, the argument given in Sec. III to show that $\Psi=\Phi$ is not affected by such an ambiguity either. The reason is that the ambiguous extra terms for $\delta \mathcal{T}_{i j}$ with $i \neq j$ vanish because both $\left\langle\hat{T}_{\mu \nu}^{(0)}\right\rangle_{\text {ren }}$ (or $\mathcal{T}_{\mu \nu}$ ) and the scalar metric perturbations in the longitudinal gauge are diagonal. Similarly, each one of the terms appearing in the $0 i$ component of the Einstein-Langevin and the quantum version of the linearized Einstein equations considered in detail in Secs. III B and III C, respectively, do not suffer from the ambiguity either.

The tensors $G_{a b}^{(1)}$ and $T_{a b}^{(1)}$, which are discussed above, result from linearizing just the metric perturbations. On the other hand, in the usual treatment of cosmological perturbations in inflationary models not only the metric perturbations but also the inflaton perturbations are simultaneously linearized. Therefore, we introduced the notation $\mathcal{T}_{a b}$ for the contribution to the expectation value of the background stress tensor $\left\langle\hat{T}_{a b}^{(0)}\right\rangle_{\text {ren }}$ which is quadratic in the background solution $\phi(\eta)$ and independent of the inflaton perturbations, i.e., the first term on the right-hand side of Eq. (12): $\mathcal{T}_{a b}=\left\langle\hat{T}_{a b}^{(0)}\right\rangle_{\phi \phi}$. As we already explained in Sec. II B, the expectation value $\left\langle\hat{T}_{a b}^{(0)}\right\rangle_{\text {ren }}$ has also a contribution which is quadratic in the inflaton perturbations but it is neglected when linearizing in those. We also introduced the notation $\delta \hat{\mathcal{T}}_{a b}$ for the perturbed stress tensor operator obtained when linearizing with respect to both the metric perturbations and the inflaton perturbations. Its expectation value $\left\langle\delta \hat{\mathcal{T}}_{a b}[g+h]\right\rangle$ is, thus, equivalent to linearizing also with respect to the inflaton perturbations the expectation value $\left\langle\hat{T}_{a b}^{(1)}[g+h]\right\rangle_{\text {ren }}$. Similarly, the operator $\delta \hat{\mathcal{T}}_{a b}[g+h]$ corresponds to linearizing in the inflaton perturbations the expression $\hat{T}_{a b}^{(0)}-$
$\left\langle\hat{T}_{a b}^{(0)}\right\rangle+\hat{T}_{a b}^{(1)}$. This expression, which appears (after raising one index) in Eq. (26), might seem a bit awkward at that point, but this is just because the notation generally employed for the Einstein-Langevin equation, where one linearizes only with respect to the metric perturbations, is no longer the most natural when one also linearizes with respect to the inflaton perturbations. The expression is appropriate either when quantizing both the metric perturbations and the scalar field perturbations or when considering a stochastic version of it, namely, the EinsteinLangevin equation. In the latter case one takes the expectation value of $\hat{T}_{a b}^{(1)}$ plus a stochastic source that accounts for the quantum fluctuations of the operator $\hat{t}_{a b}=\hat{T}_{a b}^{(0)}-$ $\left\langle\hat{T}_{a b}^{(0)}\right\rangle$, whose expectation value vanishes. Finally, the notation $\delta \hat{t}_{a b}$ is used for the result of linearizing the operator $\hat{t}_{a b}$ with respect to the inflaton perturbations, which coincides with $\delta \hat{\mathcal{T}}_{a b}$ evaluated on the unperturbed metric.

\section{APPENDIX C: FUNCTIONAL APPROACH TO THE EINSTEIN-LANGEVIN EQUATION AND ALTERNATIVE DERIVATION OF EQ. (52)}

The Einstein-Langevin equation for metric perturbations around a given background and interacting with quantum matter fields has been formally derived using functional methods [54,56-60,62]. This was achieved by regarding the metric perturbations as an open quantum system with the environment corresponding to the quantum matter fields, and using the influence functional formalism for open quantum systems introduced by Feynman and Vernon $[34,35]$. In this appendix we briefly review some basic aspects of the functional approach to the EinsteinLangevin equation and explain how an alternative derivation of Eq. (52) for the expectation value of the linearized stress tensor operator evaluated on the perturbed metric can be obtained.

When considering derivations of the Einstein-Langevin equation using functional methods, one begins by computing the influence functional for the metric perturbations by integrating out the quantum matter fields (we will only consider free fields) as follows:

$$
\begin{aligned}
e^{i S_{\mathrm{IF}}\left[h, h^{\prime}\right]}= & \int d \varphi_{\mathrm{f}} d \varphi_{\mathrm{i}} d \varphi_{\mathrm{i}}^{\prime} \int_{\varphi\left(t_{\mathrm{i}}\right)=\varphi_{\mathrm{i}}}^{\varphi\left(t_{\mathrm{f}}\right)=\varphi_{\mathrm{f}}} \mathcal{D} \varphi \\
& \times \int_{\varphi^{\prime}\left(t_{\mathrm{i}}\right)=\varphi_{\mathrm{i}}^{\prime}}^{\varphi^{\prime}\left(t_{\mathrm{f}}\right)=\varphi_{\mathrm{f}}} \mathcal{D} \varphi^{\prime} e^{i S[\varphi, g+h]-i S\left[\varphi^{\prime}, g+h^{\prime}\right]} \\
& \times \rho\left[\varphi_{\mathrm{i}}, \varphi_{\mathrm{i}}^{\prime} ; t_{\mathrm{i}}\right),
\end{aligned}
$$

where $\rho\left[\varphi_{\mathrm{i}}, \varphi_{\mathrm{i}}^{\prime} ; t_{\mathrm{i}}\right)$ is the density matrix for the initial state of the matter field $\varphi$, which is assumed to be initially uncorrelated with the metric perturbations (moreover, asymptotic initial conditions with $t_{\mathrm{i}} \rightarrow-\infty$ are usually considered) and $S[\varphi, g+h]$ is the action for the matter field evolving on a spacetime with metric $g_{a b}+h_{a b}$. Furthermore, only terms up to quadratic order in the metric 
perturbations $h_{a b}$ around the fixed background metric $g_{a b}$ will be considered. In that case the action for the matter field can be written as

$$
\begin{aligned}
S[\varphi, g+h]= & \frac{1}{2} \int d^{4} x \sqrt{-g(x)} h_{a b}(x) T^{a b}\left[\varphi, g_{a b} ; x\right)+\frac{1}{4} \int d^{4} x \sqrt{-g(x)} \int d^{4} y \sqrt{-g(y)} h_{a b}(x) h_{c d}(y) \\
& \times \frac{1}{\sqrt{-g(x)} \sqrt{-g(y)}} \frac{\delta\left(\sqrt{-g(x)} T^{a b}\left[\varphi, g_{a b} ; x\right)\right)}{\delta g_{c d}(y)}+O\left(h_{a b}^{3}\right),
\end{aligned}
$$

where $\quad T^{a b}\left[\varphi, g_{a b}^{\prime} ; x\right)=2(-g(x))^{-1 / 2} \delta S[\varphi, g] / \delta g_{a b}(x)$ corresponds to the stress tensor for the matter field, whose functional derivative $(-g(y))^{-1 / 2} \delta T^{a b}\left[\varphi, g_{a b} ; x\right) / \delta g_{c d}(y)$ is a local object, i.e., proportional to the covariant delta function $(-g(y))^{-1 / 2} \delta^{(4)}(x-y)$. The influence action to quadratic order in the metric perturbations, which can be obtained by integrating out the matter field $\varphi$ as explained in Refs. [54,62], exhibits a structure analogous to that of a linear open quantum system:

$$
S_{\mathrm{IF}}[\Sigma, \Delta]=Z \cdot \Delta+\Delta \cdot(H+K) \cdot \Sigma+\frac{i}{8} \Delta \cdot N \cdot \Delta,
$$

where $A \cdot B$ denotes $\int d^{4} x \sqrt{-g(x)} A_{a b}(x) B^{a b}(x)$ and we introduced the average and difference variables $\Sigma_{a b}=$ $\left(h_{a b}+h_{a b}^{\prime}\right) / 2$ and $\Delta_{a b}=h_{a b}^{\prime}-h_{a b}$. The expressions for the kernels are the following:

$$
\begin{aligned}
& Z^{a b}(x)=-\frac{1}{2}\left\langle\hat{T}^{a b}\left[\hat{\varphi}, g_{a b} ; x\right)\right\rangle, \\
& H^{a b c d}(x, y)=\frac{1}{4} \operatorname{Im}\left\langle T^{*} \hat{T}^{a b}[\hat{\varphi}, g ; x) \hat{T}^{c d}[\hat{\varphi}, g ; y)\right\rangle \\
& -\frac{i}{8}\left\langle\left[\hat{T}^{a b}[\hat{\varphi}, g ; x), \hat{T}^{c d}[\hat{\varphi}, g ; y)\right]\right\rangle, \\
& K^{a b c d}(x, y)=\frac{-1}{2 \sqrt{-g(x)} \sqrt{-g(y)}} \\
& \times \frac{\delta\left(\sqrt{-g(x)}\left\langle\hat{T}^{a b}\left[\hat{\varphi}, g_{a b} ; x\right)\right\rangle\right)}{\delta g_{c d}(y)}, \\
& N^{a b c d}(x, y)=\frac{1}{2}\left\langle\left\{\hat{t}^{a b}[\hat{\varphi}, g ; x), \hat{t}^{c d}[\hat{\varphi}, g ; y)\right\}\right\rangle,
\end{aligned}
$$

where the functional derivative in Eq. (C6) should be understood to account only for the explicit dependence on the metric, whereas the implicit dependence through the field operator $\hat{\varphi}[g]$ is not included. The notation $T^{*}$ appearing in Eq. (C5) means that the matter field operators must be temporally ordered before applying any derivatives acting on them. Thus, we have, for instance, $\left\langle T^{*} \nabla_{a}^{x} \hat{\varphi}(x) \nabla_{b}^{y} \hat{\varphi}(y)\right\rangle=\nabla_{a}^{x} \nabla_{b}^{y}\langle T \hat{\varphi}(x) \hat{\varphi}(y)\rangle$. Note that although the background geometry is nontrivial in general, the notion of temporal ordering is well-defined because we are restricting the possible background geometries to globally hyperbolic spacetimes, which are time orientable; moreover, the microcausality condition of the quantum field theory for the matter fields under consideration guarantees that $\left\langle\left[\hat{O}_{1}(x), \hat{O}_{2}(y)\right]\right\rangle=0$ if $\hat{O}_{1}(x)$ and $\hat{O}_{2}(y)$ are local operators and $x$ and $y$ are spacelike separated points. It should also be noted that the first term on the right-hand side of Eq. (C5) is symmetric under interchange of $x$ and $y$, whereas the second one is completely antisymmetric. On the other hand, the term on the right-hand side of Eq. (C6) is local and symmetric under interchange of $x$ and $y$.

The noise kernel $N^{a b c d}(x, y)$ requires no renormalization, as explained in Sec. II A, whereas the kernels $Z^{a b}(x)$, $H^{a b c d}(x, y)$ and $K^{a b c d}(x, y)$ contain divergences [some regularization procedure is implicitly understood in Eqs. (C4)-(C7)] that can be canceled out by adding suitable counterterms, quadratic in the curvature, to the bare gravitational action. These are precisely the same counterterms which are introduced in semiclassical gravity so that, when functionally differentiating with respect to the metric, they cancel the divergences from the expectation value of the stress tensor. This fact should not be surprising at all since the kernel $Z^{a b}(x)$ corresponds to the expectation value of the stress tensor operator on the background metric and the kernels $H^{a b c d}(x, y)$ and $K^{a b c d}(x, y)$ are closely related to the expectation value of the stress tensor operator on the perturbed metric, as follows straightforwardly from the following relation, valid through linear order in $h_{a b}$ :

$$
\begin{aligned}
\left\langle\hat{T}^{a b}[g+h ; x)\right\rangle= & \left.\frac{2}{\sqrt{-(g+h)(x)}} \frac{\delta S_{\mathrm{IF}}\left[h, h^{\prime}\right]}{\delta h_{a b}(x)}\right|_{h^{\prime}=h} \\
= & -2 Z^{a b}(x)-2(H \cdot h)^{a b}(x) \\
& -2(M \cdot h)^{a b}(x),
\end{aligned}
$$

where we introduced the kernel $M^{a b c d}(x, y)$ defined as follows:

$$
M^{a b c d}(x, y) \equiv \frac{-1}{2 \sqrt{-g(y)}} \frac{\delta\left(\left\langle\hat{T}^{a b}\left[\hat{\varphi}, g_{a b} ; x\right)\right\rangle\right)}{\delta g_{c d}(y)},
$$

which results from adding to the kernel $K^{a b c d}(x, y)$ the term $-(-g(x))^{-1 / 2}\left(\delta \sqrt{-g(x)} / \delta g_{c d}(y)\right) Z^{a b}$ coming from the contribution to the factor $2[-(g+h)(x)]^{-1 / 2}$ that is linear in $h_{a b}$. When the counterterms introduced in the bare gravitational action are included in the influence action, so that the divergences cancel out and the bare kernels $H^{a b c d}(x, y)$ and $M^{a b c d}(x, y)$ get renormalized, Eq. (C8) 
becomes

$$
\begin{aligned}
\left\langle\hat{T}^{a b}[g+h ; x)\right\rangle_{\mathrm{ren}}= & \left.\frac{2}{\sqrt{-(g+h)(x)}} \frac{\delta S_{\mathrm{IF}}^{(\mathrm{ren})}\left[h, h^{\prime}\right]}{\delta h_{a b}(x)}\right|_{h^{\prime}=h} \\
= & \left\langle\hat{T}^{a b}[g ; x)\right\rangle_{\mathrm{ren}}-2\left(H_{\mathrm{ren}} \cdot h\right)^{a b}(x) \\
& -2\left(M_{\mathrm{ren}} \cdot h\right)^{a b}(x),
\end{aligned}
$$

which can be rewritten as

$$
\left\langle\hat{T}^{(1) a b}[g+h ; x)\right\rangle_{\text {ren }}=-2\left(H_{\text {ren }} \cdot h\right)^{a b}(x)-2\left(M_{\text {ren }} \cdot h\right)^{a b}(x),
$$

where, as mentioned above, the functional derivative appearing in the kernel $M^{a b c d}(x, y)$ should be understood to account only for the explicit dependence of the stress tensor on the metric, whereas the implicit dependence through the field operator $\hat{\varphi}[g]$ is entirely contained in the first term on the right-hand side of Eq. (C11).

Taking into account the previous results, the EinsteinLangevin equation can then be obtained from the closedtime-path (CTP) effective action for the metric perturbations by using a formal trick described below. Such a CTP effective action for the metric perturbations has the following form at tree level (note, however, that the matter fields, which have already been integrated out, were treated beyond the tree level):

$$
\Gamma_{\mathrm{CTP}}^{(0)}\left[h, h^{\prime}\right]=S_{\mathrm{g}}[h]-S_{\mathrm{g}}\left[h^{\prime}\right]+S_{\mathrm{IF}}^{(\mathrm{ren})}\left[h, h^{\prime}\right]+O\left(h_{a b}^{3}\right),
$$

where $S_{\mathrm{g}}[h]$ is the Einstein-Hilbert action $\int d^{4} x \sqrt{-g} R$ through quadratic order in the metric perturbations, and the finite parts of the local counterterms have been included in $S_{\mathrm{IF}}^{(\mathrm{ren})}$. On the other hand, using the following mathematical identity for the imaginary part of the influence action:

$$
\begin{aligned}
e^{-\operatorname{Im} S_{\mathrm{IF}}} & =e^{-(1 / 8) \Delta \cdot N \cdot \Delta} \\
& =\operatorname{det}(2 \pi N)^{-(1 / 2)} \int \mathcal{D} \xi e^{-(1 / 2) \xi \cdot N^{-1} \cdot \xi} e^{-(i / 2) \xi \cdot \Delta},
\end{aligned}
$$

and interpreting $\xi^{a b}$ as a stochastic source with vanishing expectation value and correlation function $\left\langle\xi^{a b}(x) \xi^{c d}(y)\right\rangle_{\xi}=N^{a b c d}(x, y)$, one can define a stochastic effective action,

$$
\begin{aligned}
\Gamma_{\text {stoch }}[\Sigma, \Delta]= & S_{\mathrm{g}}[h]-S_{\mathrm{g}}\left[h^{\prime}\right]+\Delta \cdot\left(H_{\text {ren }}+M_{\text {ren }}\right) \cdot \Sigma \\
& -\frac{1}{2} \xi \cdot \Delta,
\end{aligned}
$$

such that $\left\langle\exp \left(i \Gamma_{\text {stoch }}\right)\right\rangle_{\xi}=\exp \left(i \Gamma_{\mathrm{CTP}}^{(0)}\right)$. The EinsteinLangevin equation can be immediately obtained by functionally differentiating with respect to the metric perturbation $h_{a b}$ and letting $h_{a b}^{\prime}=h_{a b}$ afterwards:

$$
\begin{aligned}
0= & \left.\frac{1}{\sqrt{-(g+h)(x)}} \frac{\delta \Gamma_{\text {stoch }}}{\delta h_{a b}(x)}\right|_{h^{\prime}=h} \\
= & -\frac{1}{2 \kappa} G^{(1) a b}[g+h ; x)-\left(H_{\text {ren }} \cdot h\right)^{a b}(x) \\
& -\left(M_{\text {ren }} \cdot h\right)^{a b}(x)+\frac{1}{2} \xi^{a b}(x) .
\end{aligned}
$$

It is worth discussing the issue of causality in Eq. (C15), which basically amounts to considering the second term on the right-hand side of the equation since the remaining terms are local. The right-hand side of Eq. (C5) can be formally rewritten as

$$
-\frac{i}{4}\left\langle\left[\hat{t}^{a b}[\hat{\varphi}, g ; x), \hat{t}^{c d}[\hat{\varphi}, g ; y)\right]\right\rangle \theta^{*}\left(\eta_{x}-\eta_{y}\right),
$$

where $\eta_{x}$ and $\eta_{y}$ can be any pair of well-behaved time coordinates for the points $x$ and $y$, and the star index in the theta function was used to indicate that the derivative operators acting on the scalar field which appear in the stress tensor operator should also act on the theta function. Thus, all the terms in expression (C16) are either proportional to $\theta\left(\eta_{x}-\eta_{y}\right), \delta\left(\eta_{x}-\eta_{y}\right)$, or $\delta^{\prime}\left(\eta_{x}-\eta_{y}\right)$ and, being proportional to a commutator, expression (C16) vanishes for spacelike separated points because of the microcausality condition of the quantum field theory for the matter fields. Furthermore, since both the divergences and the counterterms are local (proportional to delta functions or derivatives of them), the contribution to Eq. (C15) from the term $\left(H_{\text {ren }} \cdot h\right)_{a b}(x)$ is causal, i.e., it only depends on the metric perturbations $h_{c d}(y)$ at any point within the past lightcone of $x$.

Finally, taking into account Eq. (C11), Eq. (C15) becomes

$$
G_{a b}^{(1)}[g+h]=\kappa\left\langle\hat{T}_{a b}^{(1)}[g+h]\right\rangle_{\text {ren }}+\kappa \xi_{a b},
$$

where the indices have been lowered using the background metric. It should be noted that, in contrast to Sec. II A, the tensors appearing in Eq. (C17) correspond to perturb the background tensors with both indices already raised. However, as pointed out in Appendix B, the resulting equations in both cases are equivalent because the unperturbed tensors satisfy the semiclassical Einstein equation. Therefore, Eq. (C17) is in complete agreement with Eq. (7), keeping in mind that the finite contributions of the counterterms, corresponding to $A_{a b}$ and $B_{a b}$ in Eq. (4), have been reabsorbed in the renormalized expectation value of the stress tensor operator.

After this brief review of the functional approach to the Einstein-Langevin equation, let us now see how Eq. (C11) gives a result for $\left\langle\delta \hat{\mathcal{T}}_{0}^{i}\right\rangle_{\Phi}$ which is equivalent to that obtained in Sec. III B. To begin with, it should be pointed out that the ambiguity mentioned in Appendix B does not affect the $0 i$ component of $\left\langle\delta \hat{\mathcal{T}}_{a}^{b}\right\rangle_{\Phi}$ since both the background stress tensor and the scalar metric perturbations in 
the longitudinal gauge are diagonal. Furthermore, it can be seen that for a diagonal perturbed metric the $0 i$ component of the second term on the right-hand side of Eq. (C11) vanishes. Thus, we can concentrate on the first term.

Fourier transforming the spatial coordinates as done in Sec. III, the expectation value for the $0 i$ component of the perturbed stress tensor becomes

$$
\begin{aligned}
\left\langle\left(\hat{T}_{0 i}^{(1)}\right)_{k}[g+h]\right\rangle(\eta)= & -2 \int \sqrt{-g\left(\eta^{\prime}\right)} d \eta^{\prime} H_{0 i c d}\left(\eta, \eta^{\prime} ; \vec{k}\right) \\
& \times h_{k}^{c d}\left(\eta^{\prime}\right),
\end{aligned}
$$

where the kernel $H_{a b c d}\left(\eta, \eta^{\prime} ; \vec{k}\right)$ corresponds to the Fourier transform of the two terms on the right-hand side of Eq. (C5). Using the equivalent expression in Eq. (C16), the kernel $H_{a b c d}\left(\eta, \eta^{\prime} ; \vec{k}\right)$ is given by the following expression, which already takes into account that the Fourier transform of the expectation value $\left\langle\left[\hat{t}_{a b}[g ; \eta, \vec{x}), \hat{t}_{c d}\left[g ; \eta^{\prime}, \vec{x}^{\prime}\right)\right]\right\rangle$ is proportional to a Dirac delta function due to the existing translation invariance in the spatial coordinates:

$$
\begin{aligned}
& H_{a b c d}\left(\eta, \eta^{\prime} ; \vec{k}\right)(2 \pi)^{3} \delta\left(\vec{k}+\vec{k}^{\prime}\right) \\
& \quad=-\frac{i}{4}\left\langle\left[\left(\hat{t}_{a b}\right)_{k}[g ; \eta),\left(\hat{t}_{c d}\right)_{k^{\prime}}\left[g ; \eta^{\prime}\right)\right]\right\rangle \theta^{*}\left(\eta-\eta^{\prime}\right),
\end{aligned}
$$

where the star in the theta function had been introduced earlier to indicate that the derivatives appearing in $\hat{t}_{a b}$ and $\hat{t}_{c d}$ should also act on the theta function. Performing a similar decomposition to that introduced for the noise kernel in Eq. (20), we obtain two nonvanishing contributions to the expectation value $\left\langle\left[\hat{t}_{a b}[x ; x), \hat{t}_{c d}\left[g ; x^{\prime}\right)\right]\right\rangle[g]$ :

$$
\begin{aligned}
\left\langle\left[\hat{t}_{a b}[g ; x), \hat{t}_{c d}\left[g ; x^{\prime}\right)\right]\right\rangle= & \left\langle\left[\hat{t}_{a b}[g ; x), \hat{t}_{c d}\left[g ; x^{\prime}\right)\right]\right\rangle_{\phi \varphi} \\
& +\left\langle\left[\hat{t}_{a b}[g ; x), \hat{t}_{c d}\left[g ; x^{\prime}\right)\right]\right\rangle_{\varphi \varphi},
\end{aligned}
$$

where the first contribution is quadratic in the quantum operator $\hat{\varphi}[g]$ for the inflaton perturbations evolving on the unperturbed geometry, whereas the second contribution is quartic in $\hat{\varphi}[g]$. As already pointed out for the separation of the noise kernel, the fact that the conservation of the stress tensor, which is the source of the Einstein equation, is necessary to guarantee its integrability implies that both contributions to the expectation value must be separately conserved if we want to discard one of them keeping the consistency of the Einstein equation at the order that we are working, which is linear in the metric perturbations. This is indeed the case, as follows from the fact that both the background homogeneous solution $\phi(\eta)$ and the operator $\hat{\varphi}[g]$ satisfy the Klein-Gordon equation on the background spacetime.

If we keep only the first term on the right-hand side of Eq. (C20), i.e., if we take $H_{a b c d}\left(x, x^{\prime}\right)=$ $\left\langle\left[\delta \hat{t}_{a b}[g ; x), \delta \hat{t}_{c d}\left[g ; x^{\prime}\right)\right]\right\rangle \theta^{*}\left(\eta_{x}-\eta_{x}^{\prime}\right)$, which corresponds to considering the contributions to $\hat{t}_{a b}[g]$ that are linear in the inflaton perturbations and is consistent with the linearization of the inflaton perturbations that was considered in Secs. III and IV, we obtain

$$
\begin{aligned}
\left\langle\left(\delta \hat{\mathcal{T}}_{0 i}\right)_{k}(\eta)\right\rangle_{\Phi}= & -i \phi^{\prime}(\eta)\left(i k_{i}\right) \int_{\eta_{0}}^{\eta} d \eta^{\prime} a^{2}\left(\eta^{\prime}\right) \Phi_{k}\left(\eta^{\prime}\right) \\
& \times\left\{4 \phi^{\prime}\left(\eta^{\prime}\right)\left\langle\left[\hat{\varphi}_{k}(\eta), \hat{\varphi}_{-k}^{\prime}\left(\eta^{\prime}\right)\right]\right\rangle\right. \\
& -2 m^{2} a^{2}\left(\eta^{\prime}\right) \phi\left(\eta^{\prime}\right) \\
& \left.\times\left\langle\left[\hat{\varphi}_{k}(\eta), \hat{\varphi}_{-k}\left(\eta^{\prime}\right)\right]\right\rangle\right\} \theta^{*}\left(\eta, \eta^{\prime}\right),
\end{aligned}
$$

where we used the explicit expressions for the components $H_{0 i 00}$ and $H_{0 i j j}$, and the field $\hat{\varphi}$ corresponds to $\hat{\varphi}[g]$ here and throughout this appendix. In this case, there is actually no need for the $*$ prescription in $\theta^{*}\left(\eta, \eta^{\prime}\right)$, which implies that the derivative acting on $\hat{\varphi}\left(\eta^{\prime}\right)$ should also act on the theta function, since it yields a term proportional to $\left[\hat{\phi}_{k}(\eta), \hat{\phi}_{-k}(\eta)\right] \delta\left(\eta-\eta^{\prime}\right)$, which vanishes. Integrating by parts the first term in the integrand and using the Klein-Gordon equation for the background solution $\phi(\eta)$, given by Eq. (17), we finally get

$$
\begin{aligned}
\left\langle\left(\delta \hat{\mathcal{T}}_{0}^{i}\right)_{k}(\eta)\right\rangle_{\Phi}= & i a^{-2}(\eta) \phi^{\prime}(\eta)\left(i k_{i}\right) \int_{\eta_{0}}^{\eta} d \eta^{\prime} a^{2}\left(\eta^{\prime}\right) \\
& \times\left\{4\left[\hat{\varphi}_{k}(\eta), \hat{\varphi}_{-k}\left(\eta^{\prime}\right)\right] \phi^{\prime}\left(\eta^{\prime}\right) \Phi_{k}^{\prime}\left(\eta^{\prime}\right)\right. \\
& -2\left[\hat{\varphi}_{k}(\eta), \hat{\varphi}_{-k}\left(\eta^{\prime}\right)\right] \\
& \left.\times m^{2} a^{2}\left(\eta^{\prime}\right) \phi\left(\eta^{\prime}\right) \Phi_{k}\left(\eta^{\prime}\right)\right\},
\end{aligned}
$$

where the factor $a^{-2}(\eta)$ comes from raising the index $i$ with the background metric and we have substituted the expectation value for the commutator of the field operators simply by the commutator since for a linear theory they are $c$-numbers, whose expectation value is independent of the state. This result for the expectation value of the stress tensor coincides with Eq. (52), found in Sec. III B. It should be noted that the contribution from the boundary term at $\eta^{\prime}=\eta$ which results from the integration by parts vanishes because $\left[\hat{\varphi}_{k}(\eta), \hat{\varphi}_{-k}(\eta)\right]=0$. On the other hand, there is a nonvanishing contribution from the boundary term at $\eta^{\prime}=\eta_{0}$ :

$$
-4 k_{i} \phi^{\prime}(\eta) \phi^{\prime}\left(\eta_{0}\right)\left(\frac{a\left(\eta_{0}\right)}{a(\eta)}\right)^{2}\left[\hat{\varphi}_{k}(\eta), \hat{\varphi}_{-k}\left(\eta_{0}\right)\right] \Phi_{k}\left(\eta_{0}\right) .
$$

It might seem that the existence of this term would imply a conflict between the result for the expectation value of the linearized stress tensor operator obtained in Sec. III B using the equations of motion for the quantum operators in the Heisenberg picture and the result based on the influence functional formalism derived in this appendix. However, this is not the case. The reason for the apparent discrepancy is the following. When computing the expectation value of the stress tensor operator, there are terms proportional to 
$\langle\hat{\varphi}[g ; \eta)\rangle$, where the operator $\hat{\varphi}[g ; \eta)$, which satisfies the Klein-Gordon equation, can be written as a linear combination of a term proportional to $\hat{\varphi}\left[g ; \eta_{0}\right)$ and a term proportional to $\hat{\varphi}^{\prime}\left[g ; \eta_{0}\right)$. In particular, in Sec. III we chose a state for which both $\left\langle\hat{\varphi}\left[g ; \eta_{0}\right)\right\rangle$ and $\left\langle\hat{\varphi}^{\prime}\left[g ; \eta_{0}\right)\right\rangle$ vanished. On the other hand, in the approach based on the influence functional formalism the operators which naturally determine $\hat{\varphi}[g ; \eta)$ in terms of the initial state are $\hat{\varphi}\left[g ; \eta_{0}\right)$ and its conjugate momentum $\hat{\pi}\left[g ; \eta_{0}\right)$. Since the coupling between the metric perturbations and the inflaton perturbations involves terms proportional to the time derivative of the inflaton perturbations, $\hat{\pi}\left[g ; \eta_{0}\right)$ will differ from $\hat{\varphi}^{\prime}\left[g ; \eta_{0}\right)$ by a term proportional to the metric perturbations at the initial time. This is precisely the origin of the term in expression (C23). Thus, the apparent discrepancy is just a consequence of the fact that in the influence functional approach it has been implicitly assumed that the initial state has vanishing $\left\langle\hat{\pi}\left[g ; \eta_{0}\right)\right\rangle$ rather than vanishing $\left\langle\hat{\varphi}^{\prime}\left[g ; \eta_{0}\right)\right\rangle$.

It is important to stress that the expression in Eq. (C22) for the expectation value of the stress tensor operator needs no renormalization. This fact can be easily understood because we are dealing with the linearized theory. Therefore, the terms involved in the computation of the expectation value of the stress tensor operator are proportional to $\langle\hat{\varphi}[g+h ; x]\rangle$, whereas the divergences that arise in an exact treatment (without linearizing with respect to the scalar field) are a consequence of taking the coincidence limit $x^{\prime} \rightarrow x$ in terms involving products of the field operator, i.e., proportional to $\left\langle\hat{\varphi}[g+h ; x] \hat{\varphi}\left[g+h ; x^{\prime}\right]\right\rangle$. Alternatively, when considering Eq. (C8) together with Eq. (C16), the need for renormalization can be understood as follows. The expectation value of the commutator is finite as long as one restricts to $x \neq x^{\prime}$ but it diverges when one considers the coincidence limit. Nevertheless, it is still meaningful as a distribution. In this context, the divergences arise because the product of distributions in Eq. (C16) is ill-defined in general although each factor is welldefined as a distribution; see Ref. [92] for a detailed discussion on this point. In fact, the terms in Eq. (C16) involve terms proportional to the imaginary part of the product of two Feynman propagators $G_{F}\left(x, x^{\prime}\right) G_{F}\left(x, x^{\prime}\right)$ [55,59,93]. Working in Fourier space for the spatial variables, this product becomes $\int d^{3} q G_{F}\left(\eta, \eta^{\prime} ; \vec{k}-\vec{q}\right) G_{F}\left(\eta, \eta^{\prime} ; \vec{q}\right)$, which exhibits an ultraviolet divergence when performing the integral $\int d^{3} q$ over all possible momenta. On the other hand, when linearizing with respect to the scalar field, the Fourier-transformed version of the terms in Eq. (C16) is simply proportional to $G_{F}\left(\eta, \eta^{\prime} ; \vec{k}\right)$, with no integral over momenta and, hence, no ultraviolet divergence.

\section{APPENDIX D: INITIAL CONDITIONS}

In this appendix we will explain why, strictly speaking, a homogeneous solution $\Phi_{k}^{(\mathrm{h})}(\eta)$ with some particular initial conditions must be added to the purely inhomogeneous solution $\Phi_{k}^{(\mathrm{i})}(\eta)$ when solving the Einstein-Langevin equation as done in Secs. III and IV. The situation is completely analogous when solving the quantum version of the linearized Einstein equation as in Sec. III C.

It is well-known that the Bianchi identity guarantees the integrability of the Einstein equation provided that the stress tensor of the matter sources is covariantly conserved. Let us, however, discuss this point in some more detail. The ten components of the Einstein equation for a globally hyperbolic spacetime, which can be foliated with a set of Cauchy hypersurfaces, can be formulated as an initial value problem with time corresponding to some continuous variable labeling the Cauchy hypersurfaces. In particular for the cosmological problem that we are considering we can choose the homogeneous spatial sections labeled by the conformal time $\eta$ as the set of Cauchy hypersurfaces. The four temporal components of the Einstein equation can then be regarded as a set of dynamical constraints at any given instant of time. Thus, the integrability of the Einstein equation as an initial value problem can be understood in the following way: using the Bianchi identity and the conservation of the matter sources, the constraints can be shown to hold at any time provided that the spatial components of the Einstein equation are satisfied for all times and the four constraint equations are fulfilled on the Cauchy hypersurface corresponding to some initial time [41]. Obviously, the previous discussion can be extended to the case of the Einstein-Langevin equation since the stochastic source is also covariantly conserved.

Let us recall the temporal components of the EinsteinLangevin equation for scalar metric perturbations after Fourier transforming with respect to the spatial coordinates:

$$
\begin{gathered}
\left(k^{2}+3 \mathcal{H}^{2}\right) \Phi_{k}+3 \mathcal{H} \Phi_{k}^{\prime}=\frac{\kappa}{2} a^{2}\left(\left\langle\left(\delta \hat{\mathcal{T}}_{0}^{0}\right)_{k}\right\rangle_{\Phi}+\left(\xi_{0}^{0}\right)_{k}\right) \\
i k_{i}\left(\Phi_{k}^{\prime}+\mathcal{H} \Phi_{k}\right)=\frac{\kappa}{2} a^{2}\left(\left\langle\left(\delta \hat{\mathcal{T}}_{0}^{i}\right)_{k}\right\rangle_{\Phi}+\left(\xi_{0}^{i}\right)_{k}\right) .
\end{gathered}
$$

In Secs. III and IV the constraint equation (D2) was solved to find $\Phi_{k}(\eta)$. However, one should make sure that the remaining components of the Einstein-Langevin equation are also satisfied. According to the discussion in the previous paragraph, to make sure that this is indeed the case it is sufficient to demand that Eq. (D1) holds at the initial time $\eta_{0}$ for every $\vec{k}$. The solution of Eq. (D2) can always be written as $\Phi_{k}(\eta)=\Phi_{k}^{(\mathrm{h})}(\eta)+\Phi_{k}^{(\mathrm{i})}(\eta)$, where $\Phi_{k}^{(\mathrm{i})}(\eta)$ is a solution of the inhomogeneous equation which vanishes at the initial time $\eta_{0}$ and $\Phi_{k}^{(\mathrm{h})}(\eta)$ is a solution of the homogenous equation which is completely determined by specifying its value at $\eta_{0}$. Imposing Eq. (D1) at $\eta_{0}$ and using Eq. (D2) evaluated at $\eta_{0}$ in order to substitute $\Phi_{k}^{\prime}\left(\eta_{0}\right)$, one obtains the following result for $\Phi_{k}^{(\mathrm{h})}\left(\eta_{0}\right)$ : 


$$
\begin{aligned}
\Phi_{k}^{(\mathrm{h})}\left(\eta_{0}\right)= & \frac{\kappa}{2} a^{2}\left(\eta_{0}\right)\left(k^{2}-\frac{\kappa}{2}\left(\phi^{\prime}\left(\eta_{0}\right)\right)^{2}\right)^{-1} \\
& \times\left[\left(\xi_{0}^{0}\right)_{k}\left(\eta_{0}\right)-\frac{3 \mathcal{H}\left(\eta_{0}\right)}{i k_{i}}\left(\xi_{0}^{i}\right)_{k}\left(\eta_{0}\right)\right] \\
= & \frac{\kappa}{2} a^{2}\left(\eta_{0}\right)\left(k^{2}+\mathcal{H}^{\prime}\left(\eta_{0}\right)-\mathcal{H}^{2}\left(\eta_{0}\right)\right)^{-1} \\
& \times\left[\left(\xi_{0}^{0}\right)_{k}\left(\eta_{0}\right)-\frac{3 \mathcal{H}\left(\eta_{0}\right)}{i k_{i}}\left(\xi_{0}^{i}\right)_{k}\left(\eta_{0}\right)\right],
\end{aligned}
$$

where we took into account that $\left\langle\left(\delta \hat{\mathcal{T}}_{0}^{i}\right)_{k}\left(\eta_{0}\right)\right\rangle_{\Phi}$ vanishes, as can be immediately seen from Eqs. (52) or (C21), because the limits of integration coincide. We also used the fact that $\left\langle\left(\delta \hat{\mathcal{T}}_{0}^{0}\right)_{k}\left(\eta_{0}\right)\right\rangle_{\Phi}=a^{-2}\left(\eta_{0}\right)\left[\phi^{\prime}\left(\eta_{0}\right)\right]^{2} \Phi_{k}\left(\eta_{0}\right)$ : in this case the second term on the right-hand side of Eq. (C8) vanishes for the same reason as with $\left\langle\left(\delta \hat{\mathcal{T}}_{0}^{i}\right)_{k}\left(\eta_{0}\right)\right\rangle_{\Phi}$ but there is a nonvanishing contribution from the last term in Eq. (C8), which corresponds to the first term on the right-hand side of Eq. (29).

Since Eq. (D2) is a first-order integro-differential equation, the result for $\Phi_{k}^{(\mathrm{h})}\left(\eta_{0}\right)$ in Eq. (D3) completely determines the homogeneous solution $\Phi_{k}^{(\mathrm{h})}(\eta)$. The situation will be completely analogous when linearizing and quantizing both the metric perturbations and the inflaton perturbations, as done in Sec. III C, with the quantum operator for the metric perturbations $\hat{\Phi}(x)$ replacing the stochastic scalar field $\Phi(x)$ and the operator $\delta \hat{t}_{a}^{b}$ instead of the stochastic source $\xi_{a}^{b}(x)$. Hence, the argument concerning the equivalence between the quantum correlation function for the metric perturbations and the stochastic correlation function can be straightforwardly extended, following the same line of reasoning as in Sec. III C, to the case in which the contribution from the homogeneous solution is also taken into account.

Nevertheless, in Secs. III and IV this homogeneous solution was not considered when giving the final result for the correlation function of the metric perturbations. Therefore, we end this appendix arguing why it is justified to neglect the contribution from the homogeneous solution when computing the correlation functions for scalar metric perturbations at large scales in the context of cosmological inflationary models. In other words, the contribution from the first three terms on the right-hand side of Eq. (45) is much smaller than the contribution from the fourth term when considering a situation similar to that addressed in Sec. IV. This can be qualitatively understood in the following way. Since Eq. (D2) is a linear first-order differential equation, the solution of the homogeneous equation $\Phi_{k}^{(\mathrm{h})}(\eta)$ will be proportional to the expression for $\Phi_{k}^{(\mathrm{h})}(\eta)$ given by Eq. (D3). Thus, the first term on the right-hand side of Eq. (45) is proportional to the correlation functions for the stochastic source at the time $\eta_{0}$, and the second and third terms are proportional to the correlation functions at different times: $\eta_{0}$ and a time $\eta^{\prime}$ which is integrated from $\eta_{0}$ to $\eta_{1}$ or $\eta_{2}$ [see Eq. (44)]. The value of the noise kernel is small when one or both of the two arguments are $\eta_{0}$ provided that $\eta_{0}$ is negative enough so that the scales of interest were well inside the horizon at that time. This is in contrast to the contribution from the last term in Eq. (45) when the relevant scales are well outside the horizon at $\eta_{1}$ and $\eta_{2}$, since the two arguments of the noise kernel in that term are integrated from $\eta_{0}$ to $\eta_{1}$ or $\eta_{2}$. Hence, the reason for neglecting the first three terms on the right-hand side of Eq. (45) in this context is actually rather similar to the reason for the weak dependence on $\eta_{0}$ of the result obtained in Sec. IV when the scale $k$ is well outside the horizon at $\eta_{1}$ and $\eta_{2}$, and $\eta_{0}$ is negative enough so that $k$ is well inside the horizon at that time.

The previous argument can be made more precise if we concentrate on the particular model considered in Sec. IV. In that case, if we neglect the nonlocal term corresponding to $\left\langle\delta \hat{\mathcal{T}}_{0}^{i}\right\rangle_{\Phi}$, as done in Sec. IV, the expression for the homogenous solution is

$$
\begin{aligned}
\Phi_{k}^{(\mathrm{h})}(\eta)= & \frac{\kappa}{2} \frac{a^{3}\left(\eta_{0}\right)}{a(\eta)}\left(k^{2}+\mathcal{H}^{\prime}\left(\eta_{0}\right)-\mathcal{H}^{2}\left(\eta_{0}\right)\right)^{-1} \\
& \times\left[\left(\xi_{0}^{0}\right)_{k}\left(\eta_{0}\right)-\frac{3 \mathcal{H}\left(\eta_{0}\right)}{i k_{i}}\left(\xi_{0}^{i}\right)_{k}\left(\eta_{0}\right)\right] .
\end{aligned}
$$

The contribution from the first three terms on the righthand side of Eq. (45) can then be explicitly computed and compared to the last term, taking into account that $k \eta_{1}, k \eta_{2} \ll 1$, and $k \eta_{0} \gg 1$. In particular, the first term on the right-hand side of Eq. (45) is proportional to $(\kappa / 2)\left(m / m_{p}\right)^{2}(2 \pi)^{3} k^{-3} \delta\left(\vec{k}-\vec{k}^{\prime}\right) a^{2}\left(\eta_{0}\right) / a\left(\eta_{1}\right) a\left(\eta_{2}\right)$ and a sum of terms of order $1,\left(1 / k \eta_{0}\right)^{2}$, and $(m / H) \times$ $\left(1 / k \eta_{0}\right)^{2}$. The factor $a^{2}\left(\eta_{0}\right) / a\left(\eta_{1}\right) a\left(\eta_{2}\right)$, which is of order $k \eta_{1} k \eta_{2} /\left(k \eta_{0}\right)^{2}$, as well as $\left(1 / k \eta_{0}\right)^{2}$ and $m / H$ are much smaller than 1. It is thus clear that those contributions can be safely neglected as compared to the last term, which was found to be of order $(\kappa / 2)\left(m / m_{p}\right)^{2}(2 \pi)^{3} k^{-3} \delta\left(\vec{k}-\vec{k}^{\prime}\right)$ in Sec. IV.

Similarly, the second and third terms on the right-hand side of Eq. (45) are proportional to $(\kappa / 2)\left(m / m_{p}\right)^{2} \times$ $(2 \pi)^{3} k^{-3} \delta\left(\vec{k}-\vec{k}^{\prime}\right) a^{2}\left(\eta_{0}\right) / a\left(\eta_{1}\right) a\left(\eta_{2}\right)$ and a sum of terms of order 1 and $1 / k \eta_{0}$. Therefore, they can also be neglected as compared to the last term.

\section{APPENDIX E: ALTERNATIVE PROOF OF THE EQUIVALENCE BETWEEN STOCHASTIC AND QUANTUM CORRELATION FUNCTIONS}

In this appendix we provide an alternative proof of the equivalence between stochastic and quantum correlation functions whose key step is to show that the EinsteinLangevin equation for linearized cosmological perturbations implies Eq. (6.48) of Ref. [76].

Let us consider the Einstein-Langevin equation for scalar metric perturbations when one also linearizes with 
respect to the inflaton field, whose different components are given by Eqs. (37)-(39). We will take $\Psi=\Phi$, as justified by the discussion before Eq. (40), and work in Fourier space for the spatial coordinates. Next, we add Eq. (37), the $i=j=1$ component of Eq. (38) and (39) contracted with $F_{i} \equiv 2 m^{2} a^{2}\left(\phi / \phi^{\prime}\right)\left(k_{i} / k^{2}\right)$, which leads to the following result:

$$
\begin{aligned}
& \Phi_{k}^{\prime \prime}+2\left(\mathcal{H}-\frac{\phi^{\prime \prime}}{\phi^{\prime}}\right) \Phi_{k}^{\prime}+k^{2} \Phi_{k}+2\left(\mathcal{H}^{\prime}-\mathcal{H} \frac{\phi^{\prime \prime}}{\phi^{\prime}}\right) \Phi_{k} \\
& =\frac{\kappa}{2} a^{2}\left[\left\langle\delta \hat{\mathcal{T}}_{0}^{0}\right\rangle_{\Phi}+\left\langle\delta \hat{\mathcal{T}}_{1}^{1}\right\rangle_{\Phi}+F_{i}\left\langle\delta \hat{\mathcal{T}}_{0}^{i}\right\rangle_{\Phi}+\xi_{0}^{0}\right. \\
& \left.\quad+\xi_{1}^{1}+F_{i} \xi_{0}^{i}\right]_{k} .
\end{aligned}
$$

In deriving Eq. (E1) we made use of the following two relations

$$
\begin{aligned}
6 \mathcal{H}+2 m^{2} a^{2} \frac{\phi}{\phi^{\prime}} & =\frac{2}{\phi^{\prime}}\left(3 \mathcal{H} \phi^{\prime}+m^{2} a^{2} \phi\right) \\
& =2 \mathcal{H}-2 \frac{\phi^{\prime \prime}}{\phi^{\prime}}, \\
2 \mathcal{H}^{\prime}+2 \mathcal{H}(2 \mathcal{H} & \left.+m^{2} a^{2} \frac{\phi}{\phi^{\prime}}\right)=2 \mathcal{H}^{\prime}-2 \mathcal{H} \frac{\phi^{\prime \prime}}{\phi^{\prime}},
\end{aligned}
$$

which follow from the Klein-Gordon equation (17) for the background field $\phi$. The final step is to show that the righthand side of Eq. (E1) vanishes. In order to do so, it is convenient to consider first the Fourier-transformed version of Eqs. (29)-(31) for the linearized stress tensor. It is then straightforward to show that

$$
\left(\delta \mathcal{T}_{0}^{0}\right)_{k}+\left(\delta \mathcal{T}_{1}^{1}\right)_{k}+F_{i}\left(\delta \mathcal{T}_{0}^{i}\right)_{k}=0 .
$$

The same conclusion applies when $\varphi$ is promoted to a Heisenberg operator $\hat{\varphi}$, which implies that the first three terms on the right-hand side of Eq. (E1) cancel out. On the other hand, since $\left(\xi_{0}^{0}+\xi_{1}^{1}+F_{i} \xi_{0}^{i}\right)_{k}$ is a Gaussian stochastic process with vanishing mean, in order to prove that it vanishes it is sufficient to see that $\left\langle\left(\xi_{0}^{0}+\xi_{1}^{1}+F_{i} \xi_{0}^{i}\right)_{k} \times\right.$ $\left.(\eta)\left(\xi_{\mu}^{\nu}\right)_{k^{\prime}}\left(\eta^{\prime}\right)\right\rangle_{\xi}$, which is proportional to $\left\langle\left\{\left(\delta \hat{t}_{0}^{0}+\delta \hat{t}_{1}^{1}+\right.\right.\right.$ $\left.\left.\left.F_{i} \delta \hat{t}_{0}^{i}\right)_{k}(\eta),\left(\delta \hat{t}_{\mu}^{\nu}\right)_{k^{\prime}}\left(\eta^{\prime}\right)\right\}\right\rangle$, vanishes. Indeed, taking $\Phi=0$ in Eq. (E4) and promoting $\varphi$ to a Heisenberg operator, it follows that $\left(\delta \hat{t}_{0}^{0}+\delta \hat{t}_{1}^{1}+F_{i} \delta \hat{t}_{0}^{i}\right)_{k}=0$. Thus, the righthand side of Eq. (E1) vanishes and one is left with

$$
\Phi^{\prime \prime}+2\left(\mathcal{H}-\frac{\phi^{\prime \prime}}{\phi^{\prime}}\right) \Phi^{\prime}-\nabla^{2} \Phi+2\left(\mathcal{H}^{\prime}-\mathcal{H} \frac{\phi^{\prime \prime}}{\phi^{\prime}}\right) \Phi=0
$$

which coincides with Eq. (6.48) in Ref. [76]. Several remarks about Eq. (E5) are in order. First, the nonlocal terms associated with $\left\langle\delta \hat{\mathcal{T}}_{a}^{b}\right\rangle_{\Phi}$ are not present so that, when working in Fourier space for the spatial coordinates, one is left with an ordinary differential equation rather than an integro-differential one. Second, the equation exhibits no dependence on the stochastic source. However, the solutions of the Einstein-Langevin equation should also satisfy the constraint equations at the initial time in addition to Eq. (E5). According to the results in Appendix D, this implies a dependence on the stochastic source for the initial conditions $\Phi_{k}\left(\eta_{0}\right)$ and $\Phi_{k}^{\prime}\left(\eta_{0}\right)$, which will involve a linear combination of terms linearly proportional to the stochastic source [as given by Eq. (D3) and an analogous result for $\Phi_{k}^{\prime}\left(\eta_{0}\right)$ that can be obtained by substituting Eq. (D3) into Eq. (D2)]. The solution of the linearized Einstein-Langevin equation can then be written as $\Phi_{k}(\eta)=u_{1}(\eta) \Phi_{k}\left(\xi_{\mu}^{\nu}\left(\eta_{0}\right) ; \eta_{0}\right)+u_{2}(\eta) \Phi_{k}^{\prime}\left(\xi_{\mu}^{\nu}\left(\eta_{0}\right) ; \eta_{0}\right)$, where $u_{1}(\eta)$ and $u_{2}(\eta)$ are solutions of Eq. (E5) with initial conditions $u_{1}\left(\eta_{0}\right)=1, \quad u_{1}^{\prime}\left(\eta_{0}\right)=0 \quad$ and $u_{2}\left(\eta_{0}\right)=0$, $u_{2}^{\prime}\left(\eta_{0}\right)=1$. Such a dependence of the initial conditions on the stochastic source at the initial time is responsible for the nontrivial part of the stochastic correlation functions of $\Phi$ at later times.

If one quantizes both the linearized metric perturbation and the inflaton field, so that $\Phi$ and $\varphi$ are promoted to Heisenberg operators in Eqs. (33)-(35), one can easily conclude (proceeding analogously to the previous paragraph) that the operator $\hat{\Phi}$ for the linearized metric perturbations also satisfies Eq. (E5). Furthermore, the constraints at the initial time give the same results for $\hat{\Phi}_{k}\left(\eta_{0}\right)$ and $\hat{\Phi}_{k}^{\prime}\left(\eta_{0}\right)$ as in the stochastic case but with $\delta \hat{t}_{\mu}^{\nu}$ in place of $\xi_{\mu}^{\nu}$, so that the solutions of the quantum version of Eqs. (33)-(35) can be written as $\hat{\Phi}_{k}(\eta)=$ $u_{1}(\eta) \hat{\Phi}_{k}\left(\delta \hat{t}_{\mu}^{\nu}\left(\eta_{0}\right) ; \eta_{0}\right)+u_{2}(\eta) \hat{\Phi}_{k}^{\prime}\left(\delta \hat{t}_{\mu}^{\nu}\left(\eta_{0}\right) ; \eta_{0}\right)$. Taking into account that $\left\langle\xi_{\mu}^{\nu}[g ; x) \xi_{\rho}^{\sigma}[g ; y)\right\rangle_{\xi}=(1 / 2) \times$ $\left\langle\left\{\delta \hat{t}_{\mu}^{\nu}[g ; x), \delta \hat{t}_{\rho}^{\sigma}[g ; y)\right\}\right\rangle$, it is straightforward to see that the result for symmetrized two-point quantum correlation function $(1 / 2)\left\langle\left\{\hat{\Phi}_{k}\left(\eta_{1}\right), \hat{\Phi}_{k^{\prime}}\left(\eta_{2}\right)\right\}\right\rangle$ is equivalent to that for the stochastic correlation function $\left\langle\Phi_{k}\left(\eta_{1}\right) \Phi_{k^{\prime}}\left(\eta_{2}\right)\right\rangle_{\xi}$. This constitutes an alternative proof to that provided in Sec. IIIC of the equivalence between quantum and stochastic correlation functions for linearized cosmological perturbations.

We close this appendix by discussing a recent claim that there is a discrepancy between stochastic gravity and the standard treatment for superhorizon modes. More specifically, in Ref. [94] the Einstein-Langevin equation for linearized cosmological perturbations was solved using certain approximations and the correlation function for the gauge-invariant variable $\zeta$ was computed (this variable corresponds to the curvature perturbation in the uniform density gauge [95], which coincides with the comoving gauge for modes outside the horizon [96]). It was found that for modes outside the horizon and for a sufficiently large number of $e$-folds the correlation function was not constant in time, contrary to the standard result. This would be in conflict with the equivalence for linear perturbations that we have shown to hold in general. However, one can provide an exact argument which shows that the linearized 
Einstein-Langevin equation actually implies that for modes outside the horizon the correlation function of $\zeta$ remains constant in time. This means that the result in Ref. [94] seems to imply a problem with some of their approximations rather than a shortcoming of stochastic gravity for that regime as concluded there.

The exact argument is the following. One starts with the expression for $\zeta$ in terms of $\Phi[76,96,97]$ :

$$
\zeta=\frac{2}{3(1+w)}\left(\Phi+\mathcal{H}^{-1} \Phi^{\prime}\right)+\Phi,
$$

with $w=p / \rho$, where the relation between the background density $\rho$ and pressure $p$ and the background field $\phi$ is given by Eqs. (13) and (14) and the text after them. Differentiating Eq. (E6) with respect to the conformal time and multiplying by $(3 / 2) \mathcal{H}(1+w)$, one obtains

$$
\begin{aligned}
\frac{3(1+w)}{2} \mathcal{H} \zeta^{\prime}= & \Phi^{\prime \prime}+2\left(\mathcal{H}-\frac{\phi^{\prime \prime}}{\phi^{\prime}}\right) \Phi^{\prime} \\
& +2\left(\mathcal{H}^{\prime}-\mathcal{H} \frac{\phi^{\prime \prime}}{\phi^{\prime}}\right) \Phi,
\end{aligned}
$$

where we made use of the following relations, which can be derived from Eqs. (15) and (16),

$$
1+w=\frac{\rho+p}{\rho}=\frac{\kappa\left(\phi^{\prime}\right)^{2}}{3 \mathcal{H}^{2}}=\frac{2\left(\mathcal{H}^{2}-\mathcal{H}^{\prime}\right)}{3 \mathcal{H}^{2}},
$$

$$
\frac{d}{d \eta} \ln (1+w)=2 \frac{\phi^{\prime \prime}}{\phi^{\prime}}-2 \frac{\mathcal{H}^{\prime}}{\mathcal{H}}
$$

Note that the right-hand side of Eq. (E7) coincides with the left-hand side of Eq. (E5) except for the $\nabla^{2} \Phi$ term. This means that for modes outside the horizon, for which $\nabla^{2} \Phi$ can be neglected, the right-hand side of Eq. (E7) vanishes and the mode $\zeta_{k}$ remains constant in time.
[1] A. H. Guth, Phys. Rev. D 23, 347 (1981).

[2] A. D. Linde, Phys. Lett. 108B, 389 (1982).

[3] A. Albrecht and P. J. Steinhardt, Phys. Rev. Lett. 48, 1220 (1982).

[4] A. D. Linde, Phys. Lett. 129B, 177 (1983).

[5] A. D. Linde, Particle Physics and Inflationary Cosmology (Harwood, Academic, Chur, Switzerland, 1990).

[6] A. R. Liddle and D. H. Lyth, Cosmological Inflation and Large-Scale Structure (Cambridge University Press, Cambridge, England, 2000).

[7] V.F. Mukhanov and G. V. Chibisov, JETP Lett. 33, 532 (1981).

[8] A. H. Guth and S. Y. Pi, Phys. Rev. Lett. 49, 1110 (1982).

[9] A. D. Linde, Phys. Lett. 116B, 335 (1982).

[10] S. W. Hawking, Phys. Lett. 115B, 295 (1982).

[11] A. A. Starobinsky, Phys. Lett. 117B, 175 (1982).

[12] J. M. Bardeen, P. J. Steinhardt, and M. S. Turner, Phys. Rev. D 28, 679 (1983)

[13] G. F. Smoot et al., Astrophys. J. 396, L1 (1992).

[14] C. L. Bennett et al., Astrophys. J. Suppl. Ser.. 148, 1 (2003).

[15] H. V. Peiris et al., Astrophys. J. Suppl. Ser. 148, 213 (2003).

[16] D. N. Spergel et al., Astrophys. J. Suppl. Ser. 170, 377 (2007).

[17] S. Hollands and R. M. Wald, Gen. Relativ. Gravit. 34, 2043 (2002).

[18] L. Kofman, A. Linde, and V. Mukhanov, J. High Energy Phys. 10 (2002) 057.

[19] D. Polarski and A. A. Starobinsky, Classical Quantum Gravity 13, 377 (1996).
[20] C. Kiefer, J. Lesgourgues, D. Polarski, and A. A. Starobinsky, Classical Quantum Gravity 15, L67 (1998).

[21] C. Kiefer, D. Polarski, and A. A. Starobinsky, Int. J. Mod. Phys. D 7, 455 (1998).

[22] C. Kiefer and D. Polarski, Ann. Phys. (Leipzig) 7, 137 (1998).

[23] T. Tanaka and M. Sakagami, Prog. Theor. Phys. 100, 547 (1998).

[24] F. C. Lombardo and D. Lopez Nacir, Phys. Rev. D 72, 063506 (2005).

[25] D. Campo and R. Parentani, Phys. Rev. D 72, 045015 (2005).

[26] A. Perez, H. Sahlmann, and D. Sudarsky, Classical Quantum Gravity 23, 2317 (2006).

[27] B. L. Hu and E. Verdaguer, Classical Quantum Gravity 20, R1 (2003).

[28] B. L. Hu and E. Verdaguer, Living Rev. Relativity 7, 3 (2004).

[29] S. Weinberg, Phys. Rev. D 72, 043514 (2005).

[30] S. Weinberg, Phys. Rev. D 74, 023508 (2006).

[31] Y. Urakawa and K. Maeda, arXiv:0801.0126.

[32] A. A. Starobinsky, Phys. Lett. 91B, 99 (1980).

[33] E. Calzetta, A. Roura, and E. Verdaguer, Physica A 319, 188 (2003)

[34] R. P. Feynman and F. L. Vernon, Ann. Phys. (N.Y.) 24, 118 (1963).

[35] R. P. Feynman and A. R. Hibbs, Quantum Mechanics and Path Integrals (McGraw-Hill, New York, 1965).

[36] A. Roura and E. Verdaguer (unpublished).

[37] B. L. Hu, A. Roura, and E. Verdaguer, Phys. Rev. D 70, 044002 (2004). 
[38] B. L. Hu, A. Roura, and E. Verdaguer, Int. J. Theor. Phys. 43, 749 (2004).

[39] M. Gell-Mann and J. B. Hartle, Phys. Rev. D 47, 3345 (1993).

[40] C. W. Misner, K. S. Thorne, and J. A. Wheeler, Gravitation (Freeman, San Francisco, 1973).

[41] R. M. Wald, General Relativity (University of Chicago, Chicago, 1984).

[42] N.D. Birrell and P.C.W. Davies, Quantum Fields in Curved Space (Cambridge University Press, Cambridge, England, 1994).

[43] R. M. Wald, Quantum Field Theory in Curved Spacetime and Black Hole Thermodynamics (University of Chicago, Chicago, 1994).

[44] S. Hollands and R. M. Wald, Commun. Math. Phys. 231, 309 (2002).

[45] S. Hollands and R. M. Wald, Commun. Math. Phys. 237, 123 (2003).

[46] S. Hollands, arXiv:0705.3340.

[47] J. B. Hartle and G. T. Horowitz, Phys. Rev. D 24, 257 (1981).

[48] E. Tomboulis, Phys. Lett. 70B, 361 (1977).

[49] E.E. Flanagan and R. M. Wald, Phys. Rev. D 54, 6233 (1996).

[50] L. H. Ford, Ann. Phys. (N.Y.) 144, 238 (1982).

[51] C. I. Kuo and L. H. Ford, Phys. Rev. D 47, 4510 (1993).

[52] N. G. Phillips and B. L. Hu, Phys. Rev. D 55, 6123 (1997).

[53] R. Martín and E. Verdaguer, Phys. Lett. B 465, 113 (1999).

[54] R. Martín and E. Verdaguer, Phys. Rev. D 60, 084008 (1999).

[55] R. Martín and E. Verdaguer, Phys. Rev. D 61, 124024 (2000).

[56] E. Calzetta and B. L. Hu, Phys. Rev. D 49, 6636 (1994).

[57] B. L. Hu and A. Matacz, Phys. Rev. D 51, 1577 (1995).

[58] B. L. Hu and S. Sinha, Phys. Rev. D 51, 1587 (1995).

[59] A. Campos and E. Verdaguer, Phys. Rev. D 53, 1927 (1996).

[60] E. Calzetta, A. Campos, and E. Verdaguer, Phys. Rev. D 56, 2163 (1997).

[61] F. C. Lombardo and F. D. Mazzitelli, Phys. Rev. D 55, 3889 (1997).

[62] R. Martín and E. Verdaguer, Int. J. Theor. Phys. 38, 3049 (1999).

[63] B. L. Hu and A. Roura, Int. J. Theor. Phys. 46, 2204 (2007).

[64] A. Roura, J. Phys. A 40, 7075 (2007).

[65] B. L. Hu and A. Roura, Phys. Rev. D 76, 124018 (2007).

[66] A. Vilenkin, Phys. Rev. D 27, 2848 (1983).

[67] A. D. Linde, Phys. Lett. B 175, 395 (1986).
[68] A. Linde, D. Linde, and A. Mezhlumian, Phys. Rev. D 49, 1783 (1994).

[69] A. A. Starobinsky, in Field Theory, Quantum Gravity and Strings, edited by H. D. Vega and N. Sanchez (Springer, New York, 1986).

[70] J. M. Stewart, Classical Quantum Gravity 7, 1169 (1990).

[71] J. M. Bardeen, Phys. Rev. D 22, 1882 (1980).

[72] J. J. Halliwell and S. W. Hawking, Phys. Rev. D 31, 1777 (1985).

[73] E. M. Lifshitz, J. Phys. (Moscow) 10, 116 (1946).

[74] W. G. Unruh, arXiv:gr-qc/9802323.

[75] H. Kodama and M. Sasaki, Prog. Theor. Phys. Suppl. 78, 1 (1984).

[76] V.F. Mukhanov, H. A. Feldman, and R. H. Brandenberger, Phys. Rep. 215, 203 (1992).

[77] V.F. Mukhanov, Physical Foundations of Cosmology (Cambridge University Press, Cambridge, England, 2005).

[78] E. W. Kolb and M.S. Turner, The Early Universe (Addison-Wesley, Reading, MA, 1990).

[79] T. Padmanabhan, Structure Formation in the Early Universe (Cambridge University Press, Cambridge, England, 1993).

[80] E. Calzetta and B. L. Hu, Phys. Rev. D 52, 6770 (1995).

[81] A. Matacz, Phys. Rev. D 55, 1860 (1997).

[82] A. Matacz, Phys. Rev. D 56, R1836 (1997).

[83] E. Calzetta and S. Gonorazky, Phys. Rev. D 55, 1812 (1997).

[84] P. R. Anderson, W. Eaker, S. Habib, C. Molina-París, and E. Mottola, Phys. Rev. D 62, 124019 (2000).

[85] M. Bander and C. Itzkyson, Rev. Mod. Phys. 38, 346 (1966).

[86] T. S. Bunch, Phys. Rev. D 18, 1844 (1978).

[87] E. Mottola, Phys. Rev. D 31, 754 (1985).

[88] A. Roura and E. Verdaguer, Int. J. Theor. Phys. 38, 3123 (1999).

[89] A. Vilenkin, Phys. Rev. D 32, 2511 (1985).

[90] S. W. Hawking, T. Hertog, and H. S. Reall, Phys. Rev. D 63, 083504 (2001).

[91] C. Itzykson and J. B. Zuber, Quantum Field Theory (McGraw-Hill, New York, 1980).

[92] A. Roura and E. Verdaguer, Phys. Rev. D 60, 107503 (1999).

[93] A. Campos and E. Verdaguer, Phys. Rev. D 49, 1861 (1994).

[94] Y. Urakawa and K. Maeda, Phys. Rev. D 77, 024013 (2008).

[95] D. Wands, K. A. Malik, D. H. Lyth, and A. R. Liddle, Phys. Rev. D 62, 043527 (2000).

[96] D. H. Lyth and D. Wands, Phys. Rev. D 68, 103515 (2003).

[97] D. H. Lyth, Phys. Rev. D 31, 1792 (1985). 